\title{
Glycerol Monolaurate Attenuated Immunological Stress and Intestinal Mucosal Injury by Regulating the Gut Microbiota and AMPK/NF-KB/Nrf2 Signaling Pathway in Lipopolysaccharide-Challenged Broilers
}

\section{Linglian Kong}

Shandong Agricultural University https://orcid.org/0000-0001-7424-0582

\section{Zhenhua Wang}

Yantai University

\section{Chuanpi Xiao}

University of Liege Faculty of Gembloux Agro-Bio Tech: Universite de Liege Gembloux Agro-Bio Tech

\section{Qidong Zhu}

Shandong Agricultural University

Zhigang Song ( $\nabla$ zhigangs@sdau.edu.cn )

Shandong Agricultural University https://orcid.org/0000-0003-1525-5797

\section{Research}

Keywords: Glycerol monolaurate, Lipopolysaccharide challenge, Inflammation, Antioxidation, Gut microbiota, Adenosine monophosphate-activated protein kinase

Posted Date: November 10th, 2021

DOI: https://doi.org/10.21203/rs.3.rs-1045122/v1

License: (a) (i) This work is licensed under a Creative Commons Attribution 4.0 International License. Read Full License 


\section{Abstract \\ Background}

Antibiotic residues and resistance issues have led to the ban on antibiotic growth promoters in the poultry industry. Targeted dietary supplementation such as glycerol monolaurate (GML) has been found to ameliorate the negative effects of restriction on the use of antibiotic growth promoters by modulating the animal immune system and intestinal health. However, the mechanism by which GML contributes to the health and growth of broilers is indistinct. This study was conducted to investigate the effects of GML on immunity, intestinal barrier function, and cecal microbiota profiles in lipopolysaccharide (LPS)-challenged broilers.

\section{Results}

The results revealed that dietary GML intake augmented serum immunoglobulin A and G levels in LPSchallenged broilers. GML supplementation normalized LPS-induced variations in serum interleukin-6, interferon-y, and LPS levels; jejunal villus height; and gene expression of interleukin-6, macrophage inflammatory protein-3a, toll-like receptor 4, nuclear factor kappa-B (NF-KB), caspase-1, tight junction proteins, adenosine monophosphate-activated protein kinase a1, nuclear factor-erythroid 2-related factor 2 , and superoxide dismutase-1. GML administration ameliorated LPS-induced peroxidation by reducing malondialdehyde content and increasing antioxidant enzyme activity. Dietary GML intake enhanced the abundances of cecal probiotics such as Blautia, Lactobacillus, and Coprobacter in challenged broilers. The LPS-induced reduction in Anaerostipes, Pseudoflavonifractor, and Gordonibacter abundances in the cecum was inhibited by GML supplementation. Dietary GML intake was positively correlated with alterations in antioxidant enzyme activities and adenosine monophosphate-activated protein kinase (AMPK) a1, nuclear factor-erythroid 2-related factor 2 (Nrf2), and zonula occludens-1 levels. The genera Anaerostipes, Lachnospira, Gordonibacter, Lachnospira, Marvinbryantia, Peptococcus, and Pseudoflavonifractor were linked to attenuated inflammation and improved immunity and antioxidant capacity of LPS-challenged broilers.

\section{Conclusion}

Dietary GML intake alleviated LPS-induced immunological stress and intestinal injury in broilers. This beneficial effect of GML supplementation was attributed to the suppression of inflammation and oxidative stress by regulation of cecal microbiota and the AMPK/NF-KB/Nrf2 signaling pathway in LPS-challenged broilers.

\section{Background}

Poultry production is restricted by various environmental, nutritional, and biological pressures affecting the productive performance and health status of broilers [1]. Environmental hazards, immunological and 
oxidative stress, and bacterial infection can induce intestinal inflammation, which contribute to intestinal mucosal injury and gut epithelial dysfunction [2, 3]. Gram-negative bacterial lipopolysaccharide (LPS) is the main cause of intestinal diseases [4]. After binding to the extracellular domain of toll-like reporter 4 (TLR4), LPS initiates a cascade of intracellular signal transduction, promotes the secretion of enormous amounts of proinflammatory cytokines, and induces immunological stress. The intestinal tract is a target organ of LPS [5]. Intestinal barrier dysfunction mediated by LPS leads to increased permeability [6]. Thus, pathogenic bacteria or toxins are allowed to enter the blood circulation and cause systemic disease. Antibiotics have long been used for growth promotion and disease prevention in poultry. Nonetheless, antibiotic residues and resistance issues have pressured the poultry industry to develop substitutes for antibiotics to maintain poultry flock health [7]. Targeted dietary supplementation has been found to be conducive to modulation of the immune system and ameliorate the negative effects of environmental stressors on animal immunity and productive performance [8].

Glycerol monolaurate (GML), a monoglyceride derivative of lauric acid, possesses a large range of antimicrobial and immunomodulatory properties [9]. GML is considered an efficacious alternative to growthpromoting antibiotics and has established safety [10]. GML has been found to inhibit immune activation and the production of chemokines and cytokines by human vaginal epithelial cell cultures in vitro under the action of staphylococcal toxins [11]. GML possesses inhibitory activity against mucosal signaling and innate and inflammatory responses, protecting rhesus macaques from acute infection induced by high dosages of simian immunodeficiency virus in vivo [9]. The immunosuppressive properties of GML are conducive to attenuating intestinal inflammation [12], contributing to improved growth performance and intestinal morphology of broilers [13]. GML supplementation has been found to alleviate hyperlipidemia, hepatic steatosis, metabolic disorder, and systemic inflammation in high-fat diet (HFD)-treated mice [10]. The structure and functionality of the intestinal microbiota are crucial for the health of poultry since the gut microbiota has a strong impact on the development of the intestinal epithelium and the modulation of immunity, nutrient digestion, and intestinal barrier integrity to maintain intestinal homeostasis [14]. Dietary GML has been shown to improve performance, intestinal morphology, and muscle amino acid content in broilers by regulating the community, function, and metabolites of the intestinal microbiota [13]. The alterations of microbiota community structure modulated by GML supplementation may be the main reason for the improved intestinal health status in broilers [15].

Although the beneficial effect of GML is gradually being recognized, information on whether GML supplementation can attenuate immunological stress and protect the intestinal barrier from LPS challenge in broilers is insufficient. Significant prospects surround the microbiota in the revolutionary strategy of reducing the sensitivity of broilers to stress [16]. However, the influence of dietary GML on the intestinal microbiota in challenged broilers has not been well studied. A higher dosage of GML has been found to ameliorate chronic systemic inflammation through alteration of the intestinal microbiota in low-fat diet-fed mice [17]. Dietary GML has been shown to affect metabolism and immunity in mice and ameliorate HFDinduced metabolic disorder, obesity, and inflammation by targeting the intestinal microbiota $[18,19]$. The altered composition of the intestinal microbiota probably aggravates intestinal mucosal injury under inflammatory conditions [3]. Based on these findings, the present study tested the hypothesis that GML supplementation can alleviate the immunological stress and intestinal mucosal injury induced by LPS 
challenge through the regulation of the intestinal microbiota in broilers. Moreover, the modulatory effect of adenosine monophosphate-activated protein kinase (AMPK) in intestinal health has attracted extensive attention. Some interactions between AMPK and the intestinal microbiome have been demonstrated to regulate intestinal barrier function [20]. AMPK is an attractive target for inflammation control, as its activators inhibit the expression of nuclear factor kappa B (NF-KB) and proinflammatory cytokines induced by LPS and palmitic acid [21]. The antioxidant pathway of nuclear factor-erythroid 2-related factor 2 (Nrf2) is considered downstream of AMPK [22], which is activated to directly phosphorylate Nrf2 and inhibit oxidative stress [23]. GML possesses anti-inflammatory, antioxidant, and immunomodulatory properties [9, $15,24]$. Thus, the AMPK/NF-KB/Nrf2 signaling pathway was investigated in this study. The objective of this study was to investigate the effects of GML supplementation on growth performance, intestinal inflammation, barrier function, antioxidant status, and the cecal microbiota in LPS-challenged broilers.

\section{Methods}

\section{Animals, management and treatment}

A total of 144 one-day-old Arbor Acres broilers with similar hatching weights ( $45.7 \mathrm{~g}$ ) were randomly allocated into two dietary treatment groups as follows: basal diet and basal diet with $1200 \mathrm{mg} / \mathrm{kg} \mathrm{GML}$ (purity of more than 90\%), which was purchased from Henan Zhengtong Food Technology Co., Ltd. (Henan, China). The supplemental GML dosage was based on previous findings [15]. Each group contained 6 replicates (cages) with 12 birds per cage. The basal diet met the National Research Council 2012 recommendations for growing broilers (Table S1). All broilers were randomly assigned to metal cages $(70 \times 70 \times 40 \mathrm{~cm})$ equipped with a feeder and nipple drinker and were reared in an environmentally controlled room. The initial temperature was $34^{\circ} \mathrm{C}$ and then gradually decreased to $26^{\circ} \mathrm{C}$ by 21 days of age. The average relative humidity was maintained at approximately $70 \%$ in the first 3 days and thereafter maintained between $55 \%$ and $65 \%$. Broilers were kept under $23 \mathrm{~h}$ of light and $1 \mathrm{~h}$ of darkness in the first week, which was gradually reduced to $20 \mathrm{~h}$ and $4 \mathrm{~h}$ by the end of the 21-day experimental period.

A $2 \times 2$ two-factor completely random design was adopted in this study. Dietary GML (diet with 0 or 1200 $\mathrm{mg} / \mathrm{kg} \mathrm{GML}$ ) was considered one factor, and LPS challenge (injection with LPS or saline) was considered another. At 16, 18, and 20 days of age, half of the broilers in each replicate were intraperitoneally injected with $0.9 \%$ saline. The other half of the broilers were injected with $1 \mathrm{mg} / \mathrm{kg}$ body weight Escherichia coli LPS (L2880, Sigma-Aldrich Inc., St. Louis, MO, USA). The LPS dosage and administration procedures were applied according to existing research results [25]. On days 14 and 21, feed consumption in each replicate was recorded to calculate the average feed intake (AFI). Spilled feed was carefully collected and weighed to correct the final feed intake data. Broilers were weighed to calculate the average body weight gain (ABWG). The feed conversion ratio (FCR) was defined as AFI:ABWG.

\section{Sample collection}

Two birds per replicate were randomly selected for sampling after the growth performance was calculated at 21 days of age. Blood samples $(4 \mathrm{ml})$ were retrieved from wing veins by sterile syringes. One milliliter of blood was transferred to glass tubes coated with ethylenediaminetetraacetic acid for blood cell analysis. 
The other $3 \mathrm{ml}$ of blood samples were transferred to glass tubes without anticoagulant to separate serum by centrifugation at $3000 \mathrm{rpm}$ for $10 \mathrm{~min}$ at $4^{\circ} \mathrm{C}$. Approximately $2 \mathrm{~cm}$ segments were excised from the entry point of the bile duct to Meckel's diverticulum and immediately immersed in $4 \%$ paraformaldehyde solution for histological examination. Approximately 1-to-2 $\mathrm{g}$ jejunal samples and complete ceca were collected on ice, rapidly frozen in liquid nitrogen, and stored at $-80^{\circ} \mathrm{C}$ for further analysis.

\section{Hematology determination}

Leukocyte, lymphocyte, intermediate cell, and granulocyte counts in the blood were determined using an automatic blood counter according to the manufacturer's instructions (KT6200, Genrui Biotech Inc., Shenzhen, China).

\section{ELISA of serum and jejunal homogenate}

Intestinal permeability was evaluated based on serum diamine oxidase (DAO) and LPS levels. Interleukin 1 beta (IL-1 $\beta$ ), interleukin 6 (IL-6), tumor necrosis factor-alpha (TNF-a), interferon- $\mathrm{\gamma}$ (IFN- $\gamma$ ), immunoglobulin A $(\operatorname{IgA})$, and immunoglobulin $\mathrm{G}(\mathrm{IgG})$ levels were detected using ELISA kits to estimate the immune response status (MLBIO Co., Shanghai, China). Jejunal samples $(0.3 \mathrm{~g})$ were homogenized in $2.7 \mathrm{ml}$ of phosphatebuffered saline and centrifuged at $1000 \times \mathrm{g}$ for $10 \mathrm{~min}$ at $4^{\circ} \mathrm{C}$. Then, the supernatant was collected to detect the levels of phospho-AMPK and total AMPK by ELISA (MLBIO Co., Shanghai, China). The results were normalized to the protein concentration in each jejunal homogenate. All determination procedures were performed in strict accordance with the manufacturer's instructions. The inter- and intraassay coefficients of variation were less than $10 \%$.

\section{Jejunum morphology analysis}

The jejunum segments were dehydrated and embedded in paraffin after fixation in a $4 \%$ paraformaldehyde solution for $24 \mathrm{~h}$. Tissue coated with paraffin was sectioned at $5 \mu \mathrm{m}$ thickness using a microtome (Leica RM2235, Leica Biosystems Inc., Buffalo Grove, USA), fixed on slides, and stained with hematoxylin and eosin. Images of the jejunum were acquired using a Nikon Eclipse 80i microscope (Nikon Inc., Tokyo, Japan) and analyzed with ImageJ analysis software (version 1.47, Bethesda, MD, USA). Villus height (VH) was gauged from the tip of the villus to the villus-crypt junction. Crypt depth (CD) was defined as the depth of the invagination between adjacent villi. The villus height-to-crypt depth ratio (VCR) was calculated. Ten sections of the proper microscopic fields were selected randomly from each sample for morphology measurement. The average of ten values from individual broilers was used in statistical analysis.

\section{Assessment of oxidative status}

The oxidative status of serum and jejunal homogenate was evaluated by determining malondialdehyde (MDA) levels, total antioxidant capacity (T-AOC), catalase (CAT) capacity, and total superoxide dismutase (TSOD) activity. The protein content of jejunal homogenate was measured with a bicinchoninic acid protein assay kit. All diagnostic kits (intraassay coefficients of variation < $5 \%$, interassay coefficients of variation < 8\%) were purchased from Nanjing Jiancheng Biotechnology Institute (Nanjing, China). All determination 
procedures were performed in strict accordance with the manufacturer's instructions. The results were normalized to the protein concentration in each jejunal homogenate.

\section{RNA isolation and real-time quantitative PCR}

Jejunal RNA was isolated using RNA-Easy Isolation Reagent (Vazyme Biotech, Nanjing, China). RNA quality was evaluated using $1 \%$ agarose gel electrophoresis. The concentration of total RNA was determined by a NanoDrop 2000 spectrophotometer (Thermo Scientific, Wilmington, USA). Reverse transcription of $1 \mu \mathrm{g}$ of total RNA was performed using a PrimeScript ${ }^{\circledR}$ RT reagent kit after genomic DNA was removed by gDNA Eraser (RR047A, Takara Bio Inc., Dalian, China). $\beta$-actin, glyceraldehyde-3-phosphate dehydrogenase, and TATA-binding protein were selected as the housekeeping genes. The primer sequences are shown in Table S2. A standard curve was used to verify the amplification efficiency per pair of primers. The reversetranscribed cDNA was diluted 5 times and subjected to real-time quantitative PCR. TB Green Premix Ex Taq (RR820A, Takara Bio Inc., Dalian, China) and an Applied Biosystems 7500 real-time PCR system (Applied Biosystems, CA, USA) were used. The reaction program was as follows: predenaturation at $95^{\circ} \mathrm{C}$ for $10 \mathrm{~s}$, then a total of 40 cycles of denaturation at $95^{\circ} \mathrm{C}$ for $5 \mathrm{~s}$ and annealing and extension at $60^{\circ} \mathrm{C}$ for $34 \mathrm{~s}$. Each reaction was repeated three times. The specificity of the amplified products was verified with a melting curve. The relative expression of the target gene was analyzed through the $2^{-\Delta \Delta C t}$ method after normalization against the geometric mean of the expression of the housekeeping genes.

\section{S rRNA gene sequencing and analysis}

Total genomic DNA from cecal contents was extracted using an E.Z.N.A.® Soil DNA Kit (Omega Bio-Tek, Norcross, GA, USA) following the manufacturer's protocol. The purity and concentration of final DNA were determined with a NanoDrop 2000 spectrophotometer (Thermo Scientific, Wilmington, USA). The integrity of DNA was detected by $1 \%$ agarose gel electrophoresis. The V3-V4 hypervariable regions of bacterial $16 \mathrm{~S}$ rRNA genes were amplified with the specific primers 338F (5'-ACTCCTACGGGAGGCAGCAG-3') and 806R (5'GGACTACHVGGGTWTCTAAT-3') by a GeneAmp ${ }^{\circledR} 9700$ PCR system (Applied Biosystems, CA, USA). Amplification was conducted using the following program: $3 \mathrm{~min}$ at $95^{\circ} \mathrm{C} ; 27$ cycles of $30 \mathrm{~s}$ at $95^{\circ} \mathrm{C}, 55^{\circ} \mathrm{C}$ for $30 \mathrm{~s}$, and $72^{\circ} \mathrm{C}$ for $45 \mathrm{~s}$; and $72^{\circ} \mathrm{C}$ for $10 \mathrm{~min}$. PCRs were performed in triplicate using a $20 \mu$ mixture containing $10 \mathrm{ng}$ of template DNA. The PCR products were extracted from a $2 \%$ agarose gel, purified by an AxyPrep DNA Gel Recovery Kit (Axygen Biosciences, Union City, CA, USA), and then quantified with a QuantiFluor ${ }^{\text {TM-ST }}$ blue fluorescence quantitative system (Promega, USA). Purified amplicons were pooled in equimolar amounts and paired-end sequenced on an Illumina MiSeq PE300 platform (Illumina, San Diego, USA) following standard protocols.

Raw fastq files obtained by MiSeq sequencing were demultiplexed, quality filtered, trimmed, denoised with QIIME (version 1.9.1, http://qiime.org/install/index.html) and merged according to the overlapping relationship by FLASH (version 1.2.11, https://ccb.jhu.edu/software/FLASH/index.shtml). Sequences with 97\% similarity were clustered into the same operational taxonomic units (OTUs) and analyzed using UPARSE (version 7.1, http://www.drive5.com/uparse/). Chimeras were removed during clustering. The OTU representative sequences were analyzed with the RDP Classifier (version 2.2, 
http://sourceforge.net/projects/rdp-classifier/) against the Silva 16S rRNA database (release 119, http://www.arb-silva.de) using a confidence threshold of 70\%. Diversity metrics were calculated using the core-diversity plugin within QIIME2. The Chao1, Faith-phylogenetic diversity (faith-pd), Shannon, and Simpson indices were calculated to estimate the microbial diversity within an individual sample. $\beta$ diversity analysis based on unweighted UniFrac distance was performed to investigate the structural variation in microbial communities across samples and then visualized by principal coordinate analysis (PCoA). Linear discriminant analysis effect size (LEfSe) was employed to identify the bacteria with different abundances among samples and groups. Redundancy analysis (RDA) was performed to reveal the association of microbial communities with environmental factors. Spearman's rank correlations between predominant taxa were calculated to perform co-occurrence analysis.

\section{Statistical analysis}

All data were checked for normality with the Shapiro-Wilk test ( $95 \%$ confidence level) and subjected to twoway ANOVA using the general linear model procedure in SPSS software (SPSS 26.0, SPSS, Chicago, USA). The main factor effects and interactions of LPS challenge and dietary GML intake were evaluated. Significant variations between the treatments were compared using Tukey's multiple comparisons or nonparametric factorial Kruskal-Wallis test. Data are presented as the mean values with the total standard errors of the mean (SEMs). Differences were considered significantly different at $P<0.05$.

\section{Results}

\section{Effects of GML supplementation on growth performance}

Feed consumption and body weight were recorded to assess the impact of GML supplementation on the growth performance of LPS-challenged broilers (Table 1). The AFI and ABWG of broilers were reduced by LPS challenge $(P<0.05)$. Thus, the highest FCR was observed in the LPS-challenged group $(P<0.05)$. Dietary treatment with GML did not modify the AFI, ABWG, or FCR of broilers during the entire experimental period $(P>0.05)$. No interaction between dietary GML intake and LPS challenge was shown in the growth performance of broilers $(P>0.05)$.

\section{Effects of GML supplementation on blood cell counts}

Peripheral blood lymphocytes are sensitive to the immune response [26]. In this study, the challenged broilers had higher leukocyte, intermediate cell, and granulocyte counts in the blood than the unchallenged broilers $(P<0.05)$ (Table 2). Dietary GML decreased the leukocyte count compared to that with the control $(P<0.05)$. No interaction effect on blood cell counts was recorded after GML supplementation of LPSchallenged broilers $(P>0.05)$.

\section{Dietary GML alleviated LPS-induced cytokine production and augmented immunoglobulin levels}

GML possesses a large range of immunoregulatory properties and inhibitory activity against the production of proinflammatory cytokines [9]. To evaluate the effect of GML supplementation on the inflammatory reaction, the levels of inflammatory cytokines and immunoglobulins in the serum were detected by ELISA 
(Table 3). Significant interactions were observed between GML supplementation and LPS challenge in the serum cytokine and immunoglobulin levels of broilers $(P<0.05)$. LPS challenge increased the levels of IL$1 \beta$, IL-6, TNF- $a$, and IFN- $\gamma$ in serum $(P<0.05)$. Dietary GML reduced the serum IL-1 $\beta$ and IFN- $\gamma$ levels and reversed the increase in IL-6 and IFN- $\gamma$ levels caused by LPS challenge $(P<0.05)$. Moreover, the IgA and IgG levels were significantly increased by GML supplementation in challenged broilers $(P<0.05)$. These results indicated that the inflammatory response induced by LPS challenge was reversed by dietary GML supplementation.

\section{Dietary GML restored inflammatory gene expression in the jejunum of LPS-challenged broilers}

To further determine the genetic basis of the ameliorated inflammatory reaction in LPS-challenged broilers, the expression of genes related to cytokines and apoptosis was investigated by quantitative PCR (Table 4). The interaction between GML supplementation and LPS challenge notably affected the gene expression ofIL-6, TLR4, macrophage inflammatory protein (MIP)-3a, NF-KB, and caspase-1 in the jejunum of broilers ( $P$ $<0.05)$. LPS challenge upregulated IL-6, TLR4, MIP-3a, NF-KB, and caspase-1 expression $(P<0.05)$. Dietary GML supplementation significantly reduced jejunal MIP-3a and NF-KB expression and reversed the enhancement of proinflammatory and apoptotic gene expression mediated by LPS challenge $(P<0.05)$. The results revealed that dietary GML supplementation ameliorated jejunal inflammation and apoptosis in LPSchallenged broilers.

\section{Dietary GML enhanced jejunal VH and activated AMPK signaling}

Intestinal morphology parameters are considered an important indicator of intestinal health, recovery, and function [27]. Targeting AMPK strengthens intestinal barrier functions and exerts beneficial health impacts on the leaky gut [20]. Thus, intestinal morphology and jejunal AMPK levels were detected to investigate the effect and molecular basis of dietary GML intake on the alleviation of intestinal injury induced by LPS challenge (Table 5). A significant interaction between dietary GML intake and LPS challenge was observed in serum LPS levels, jejunal VH, and the phospho-AMPK and total AMPK contents in the jejunum of broilers $(P<0.05)$. LPS challenge led to an increase in serum DAO and LPS levels and a decrease in jejunal VH and VCR $(P<0.05)$. Dietary GML supplementation restored serum LPS levels and jejunal VH in LPSchallenged broilers $(P<0.05)$. Moreover, GML supplementation increased the jejunal phospho-AMPK and total AMPK contents compared with those of the control and LPS-treated broilers $(P<0.05)$. These results suggested that GML supplementation promoted the activation of AMPK signaling and ensured recovery of intestinal barrier function that had been injured by LPS challenge.

\section{Dietary GML restored the gene expression of tight junction proteins and AMPK in LPS-challenged broilers}

The relative gene expression of tight junction proteins and AMPK was determined to evaluate the genetic basis of improved intestinal barrier function promoted by GML supplementation. There was a significant interaction between dietary GML intake and LPS challenge $(P<0.05)$ (Table 6). GML supplementation rescued the reduction in the expression of tight junction proteins, such as zonula occludens (ZO)-1, occludin, and claudin-2, induced by LPS challenge $(P<0.05)$. Jejunal AMPKa1 and AMPKa2 expression was downregulated in the LPS-treated broilers $(P<0.05)$. An interaction based on AMPKa1 expression was 
observed between LPS challenge and dietary GML intake, and GML supplementation prevented the decrease in jejunal AMPKa1 expression in challenged broilers $(P<0.05)$. Collectively, these data suggested that the recovery of intestinal injury by GML supplementation in LPS-treated broilers may be related to the enhancement of tight junction protein expression and AMPK activation.

\section{Dietary GML intake attenuated oxidative stress induced by LPS challenge}

Since intestinal inflammation and barrier function are closely correlated with oxidative stress [28], the oxidative status in serum and the jejunum was further examined (Table 7). Significant interactions were observed between GML supplementation and LPS challenge in the oxidative status of broilers $(P<0.05)$. The LPS-challenged broilers had higher serum MDA levels than the control broilers $(P<0.05)$. Dietary GML intake reversed the MDA content elevation and CAT and T-AOC reduction induced by LPS challenge $(P<$ $0.05)$. In the jejunum, LPS challenge led to increased MDA content and reduced T-AOC $(P<0.05)$. Increased SOD and CAT activities and T-AOC were observed in the GML-treated broilers $(P<0.05)$. Dietary GML supplementation significantly increased the serum GSH-px; and jejunal SOD, CAT, T-AOC, and GSH-px activities $(P<0.05)$. These findings revealed that GML supplementation ameliorated oxidative stress in challenged broilers through the improved activity of antioxidant enzymes and maintaining the balance of oxidation and antioxidation.

\section{GML supplementation normalized the gene expression of Nrf2 and SOD1 in the jejunum of LPS-challenged broilers}

Expression of antioxidant-related genes was determined to reveal the potential mechanism by which dietary GML intake relieves oxidative stress (Table 8). LPS challenge led to the downregulation of Nrf2, SOD2, and CAT expression, which contributed to oxidative stress $(P<0.05)$. There was an interaction between dietary GML and LPS challenge in the gene expression of Nrf2 and SOD1 in the jejunum of broilers $(P<0.05)$. Dietary GML intake rescued the reduction in Nrf2 and SOD1expression induced by LPS challenge $(P<0.05)$. Moreover, although no interaction existed between dietary GML intake and LPS challenge, GML supplementation elevated jejunal heme oxygenase-1 expression in broilers $(P>0.05)$, which is considered a downstream signaling molecule of Nrf2. These data suggested that GML supplementation normalized the expression of antioxidant-related genes and ameliorated LPS-induced oxidative stress by regulating Nrf2 signaling.

\section{Effect of GML supplementation on the microbiota of the cecal lumen}

16S rRNA gene high-throughput sequencing was performed to reveal the role of the cecal microbiota in GML attenuation of the immune stress and intestinal injury promoted by LPS challenge. A Venn diagram showed that $1383,1341,1379$, and 1311 specific OTUs existed in the control (CON), GML, LPS, and LPS + GML groups, respectively, with 654 OTUs shared (Fig. 1A). The microbial diversity within an individual sample was assessed by the Chao1, Faith-pd, Shannon, and Simpson indices, with no alteration among all the groups (Fig. 1B). PCoA of the OTUs was performed to assess similarities and differences among samples and groups (Fig. 1C). The results revealed that the cecal microbiota in the LPS-challenged groups was separated from that in the CON and GML groups. The differences in species diversity were quantified by 
ANOSIM based on unweighted UniFrac distance, which indicated that the LPS challenge altered the $\beta$ diversity index compared to that of the CON and GML groups (Fig. 1D). Collectively, these results indicated that LPS challenge modulated the cecal microbiota community structure of broiler chicks. Taxonomic profiling indicated that 18 bacterial phyla were present in the cecum of broilers (Fig. 2A). Firmicutes, Bacteroidetes, and Proteobacteria accounted for most of the intestinal bacteria of broilers. Dietary GML supplementation significantly reduced the amount of the Elusimicrobia phylum in LPS-challenged broilers (Fig. 2C). The relative abundance of the top 20 families was analyzed to further explore changes in the cecal microbiota (Fig. 2B). Dietary GML intake increased the relative abundance of Lactobacillaceae and reduced the proportion of Acidaminococcaceae and Elusimicrobiaceae in the cecum of LPS-treated broilers (Fig. 2C). Furthermore, the relative abundance of the 20 predominant genera per group was analyzed to illustrate the specific changes in the microbial taxa (Fig. 3A). LEfSe (LDA score $>2$ ) was performed to detect the specific bacterial taxa associated with LPS challenge and dietary GML intake (Fig. 3B). Only the genus Marvinbryantia was notably abundant in the LPS group. Dietary GML intake significantly increased the relative abundances of cecal Blautia, Lactobacillus, and Coprobacter in challenged broilers compared with those in normal broilers (Fig. 3C). GML supplementation reversed the reduced relative abundances of Anaerostipes, Pseudoflavonifractor, and Gordonibacter induced by LPS in broilers (Fig. 3C). Administration of GML reduced the proportions of cecal Phascolarctobacterium and Elusimicrobium in the LPS-treated broilers (Fig. 3C). The abundance of Marvinbryantia was lower in the LPS+GML group than in the LPS group, with an insignificant difference (Fig. 3C). These data indicated that dietary supplementation with GML increased the abundance of cecal probiotics and reduced the population of pathogenic bacteria in LPS-challenged broilers. An obvious regulatory effect of dietary GML was observed on the cecal microorganisms of broilers under LPS challenge.

The serum GSH-px, IgA, and IgG levels in LPS-challenged broilers were positively correlated with GML supplementation (Fig. 4A). The serum LPS, MDA, and IL-6 levels and jejunal gene expression of TLR4, IL-6, and NF-KB were positively associated with LPS challenge but negatively linked to dietary GML intake (Fig. $4 \mathrm{~A})$. Moreover, the activity of jejunal antioxidant enzymes and the gene expression of AMPKa1, Nrf2, and ZO-1 were positively related to GML supplementation (Fig. 4B). The associations among the intestinal microbiota and immune indices, antioxidant enzymes, tight junction proteins, AMPK, and the NF-KB signaling pathway were further analyzed at the genus level and visualized by heatmap (Fig. 4C). The levels of inflammatory factors and NF-KB signaling were positively linked to the genera Roseburia, Marvinbryantia, GCA_900066575, and Subdoligranulum but negatively correlated with Ruminiclostridium_9 and Pseudoflavonifractor. Serum IgA and IgG levels were positively associated with Peptococcus, Lachnospira, Gordonibacter, and Lachnoclostridium abundances. Tight junction proteins and AMPK signaling were possibly correlated with Anaerostipes, Pseudoflavonifractor, and Ruminiclostridium_9 but negatively correlated with the genera Parabacteroides, Marvinbryantia, Merdibacter, and Eubacterium_hallii_group. The abundances of Pseudoflavonifractor, Peptococcus, Lachnospira, Gordonibacter, and Anaerostipes were positively associated with antioxidant capacity and jejunal Nrf2 gene expression. These results indicated that the augmented intestinal health of LPS-challenged broilers was probably linked to the altered cecal microbiota manipulated by GML supplementation. 


\section{Discussion}

Broilers are inevitably affected by immunological challenges or oxidative stress relevant to bacteria or LPS challenges [29]. Immunological stress, oxidative stress, and gastrointestinal health can influence the health status of poultry and are thus considered critical issues in poultry production [30]. LPS is widely applied to establish a model of intestinal injury mediated by immunological and oxidative stress [31]. Numerous studies have demonstrated that LPS challenge compromises the feed intake of broilers owing to LPSinduced impairment of the intestinal barrier [31,32]. This study confirmed the destructive consequences of LPS challenge on growth performance, as evidenced by decreased AFI and ABWG and increased FCR in challenged broilers. The impaired growth performance of broilers is related to suppressed appetite [33], intestinal barrier dysfunction [34], and nutrition malabsorption [32]. Under stress conditions, the partitioning of dietary nutrients is altered to support the immune system [35]. No alterations have been reported in the body weight, ABWG, or FCR of broilers supplemented with GML in both the first twenty-eight days and all experimental periods $[36,8]$. In this study, the decreased growth performance induced by LPS challenge was not rescued by GML supplementation. This result confirmed that the growth performance of broilers was insensitive to GML supplementation even under challenge conditions. Peripheral blood lymphocytes quickly respond to immune stress [26]. LPS challenge can elevate blood leukocyte, lymphocyte, mononuclear cell, and neutrophil counts [6]. Accordingly, intraperitoneal injection with LPS increased the number of leucocytes, lymphocytes, and granulocytes in the blood of broilers, which suggested that the inflammatory response was induced by LPS challenge in this study.

After specific recognition by leukocyte membrane toll-like receptors and myeloid differential proteins, LPS induces immunological and oxidative stress by stimulating the secretion of numerous cytokines, such as IL6 and IL-1 [37]. In this study, LPS challenge elevated the production of serum IL-1 $\beta$, IL-6, TNF-a, and IFN- $\gamma$ and upregulated the expression of jejunal IL-6, TLR4, MIP-3a, NF-KB, and caspase-1, which promoted immunological stress and apoptosis. GML possesses immunomodulatory properties with inhibitory activity against the production of MIP-3a and the secretion of proinflammatory cytokines [11]. In vivo, GML supplementation reduced the serum IL- 6 and TNF- $\beta$ levels and alleviated the systemic inflammatory response of HFD-fed mice [19]. In vitro, lauric acid and GML could be used to promote resolution following an inflammatory insult [38]. Accordingly, in this study, dietary GML intake moderated immunological stress promoted by LPS challenge with alleviated levels and gene expression of MIP-3a and proinflammatory cytokines. NF-KB is a central regulator of inflammatory responses involved in most innate immune receptor signaling pathways. The activation of NF-KB is considered one of the central ways that feed compounds affect immunity [38]. Fatty acid treatment has been found to reduce the activation of NF-KB in a dosedependent manner under LPS challenge compared with that in the absence of LPS [39]. In this study, dietary GML intake reduced jejunal NF-KB expression in LPS-challenged broilers, which indicated that GML-relieved inflammation may be related to regulation of the NF-KB signaling pathway. Moreover, AMPK has been implicated as an attractive target for inflammation control because activated AMPK has been shown to suppress LPS-induced NF-kB activity and the production of proinflammatory cytokines in multiple cell types [40]. In the present study, dietary GML intake increased the protein levels of total and phosphorylated AMPK in the jejunum of LPS-challenged broilers and rescued the LPS-induced downregulation of AMPKa1 expression. This phenomenon indicated that GML supplementation elevated the activation of AMPK 
signaling in LPS-challenged broilers. Induction of AMPK signaling has been shown to inhibit the expression of TNF-a, IL-6, and IL-1 $\beta$ through suppression of the nuclear translocation of NF-KB in mouse neurons [41]. Thus, the inhibited NF-KB activity in this study was conjectured to be linked to the activation of AMPK mediated by GML supplementation. These results indicated that dietary GML intake attenuated LPSinduced immunological stress by alleviating intestinal inflammation, which was probably linked to specific activation of AMPK and suppression of NF-KB activity.

Intestinal integrity is the key factor preventing infection of broilers by pathogenic bacteria [31]. DAO is considered a sensitive indicator reflecting intestinal injury [42]. In the present study, LPS challenge induced increased levels of serum DAO and LPS in broilers, which was in line with previous findings [43]. Notably, dietary GML supplementation rescued the increased serum LPS and DAO levels, which revealed an improvement in intestinal permeability and injury in LPS-challenged broilers. Reduced gene expression of tight junction proteins can affect intestinal permeability and disrupt the intestinal barrier [44]. Thus, macromolecules were able to translocate into the blood circulation from the intestinal cavity, which led to increased LPS and DAO levels in serum in LPS-treated broilers. In this study, LPS challenge downregulated the jejunal gene expression of ZO-1, occludin, and claudin-2 to varying degrees, which was reversed by GML supplementation, revealing recovery of the intestinal barrier and gut injury. Intestinal morphology reflects the health status of the gut and the response to certain feed substances [45]. In the present study, a reduction in jejunal VH and VCR and an impaired intestinal morphology were produced by LPS challenge, which was consistent with previous studies [13]. GML supplementation has been found to improve intestinal morphology $[13,15]$. In the present study, dietary GML intake increased jejunal VH and VCR, reduced intestinal CD, and reversed the LPS-induced decrease in VH of the jejunum in broilers, which indicated an augmentation of intestinal morphology. The LPS-induced impairment of intestinal morphology have been shown to be rescued by natural extracts with anti-inflammatory effects [31]. Thus, the ameliorative effect of GML on intestinal inflammation may be a potential reason for the improved intestinal integrity in challenged broilers. Moreover, AMPK has been shown to be involved in the tight junction assembly process in challenged epithelial cells, and its activation enhances recovery of epithelial barrier function following injury [20]. Intestinal AMPK activity has been notably linked to colitis development in inflammatory mouse models [46]. Butyrate, a short-chain fatty acid, promotes tight junction assembly and regulates intestinal barrier function by activating AMPK in human colon carcinoma cells [47]. In this study, dietary GML intake augmented the gene expression of tight junction proteins and activation of AMPK in LPS-challenged broilers. These results indicate that dietary GML supplementation ameliorated LPS-induced intestinal injury and barrier dysfunction by attenuating inflammation and activating AMPK signaling.

Oxidative damage in broiler tissues is promoted by LPS challenge owing to the disrupted balance between pro-oxidant and antioxidant systems [48]. The sophisticated antioxidant defense system, including enzymes T-SOD, CAT, and GSH-px, is crucial to counteract oxidative damage. These antioxidant enzymes are considered sensitive indicators to reflect oxidative stress in animals [30]. LPS has been reported to induce oxidative stress in broilers, which is characterized by decreased T-SOD, CAT, and GSH-px activities in the serum and liver [30]. In the present study, LPS challenge elevated the serum and jejunal MDA contents and reduced the activity and expression of antioxidant enzymes in the jejunum of broilers. In contrast, GML supplementation reduced MDA content and augmented the activities of antioxidant enzymes in challenged 
broilers, which revealed that LPS-induced lipid peroxidation was reversed by dietary GML intake. The peroxidation level in meat has been found to be alleviated by GML-supplied diets and to be proportional to the increase in dietary additive concentration [24]. Dietary GML intake has been shown to enhance the antioxidant capacity of laying hens by reducing MDA content and increasing SOD activity [36]. In this study, GML supplementation modulated the delicate balance between oxidants and antioxidants and alleviated oxidative stress in LPS-challenged broilers. Oxidative stress is significantly connected with inflammation. These two processes induce each other reciprocally, prompting a vicious cycle [28]. Thus, the relieving effect of dietary GML on oxidative stress induced by LPS challenge may be related to the reversal of the inflammatory response. Moreover, Nrf2 is a master regulator of cellular defenses against oxidative stress and is activated to promote the expression of antioxidant genes such as heme oxygenase-1 [49]. AMPK serves as a stress sensor and exerts a beneficial effect on the alleviation of oxidative stress [50]. AMPK has been found to directly phosphorylate Nrf2 in mouse embryonic fibroblasts [23]. In the present study, dietary GML intake rescued the reduced expression of Nrf2 and SOD1 induced by LPS challenge, which paralleled the changes in AMPK signaling. Overall, dietary GML intake exerted antioxidant properties and attenuated LPS-induced oxidative stress, probably by regulating the AMPK/Nrf2 signaling pathway and enhancing antioxidant enzyme activity.

A large number of microorganisms colonize the gastrointestinal tract of poultry and closely and densely interact with the feed and host. The gut microbiome benefits the host through the exchange of nutrients and modulation of host gut morphology, physiology, and immunity [51]. Although the intestinal microbiota itself can regulate the immune system by releasing LPS, excessive LPS levels disrupt the dynamic balance of the intestinal microbiota [52]. In the present study, the community structure of the cecal microbiota in broilers was modulated by LPS challenge, which was similar to previous findings [42,3]. The rich community of species enhances the stability of the intestinal microecology and leads to decreased sensitivity to bacterial invasion and intestinal inflammation [53]. Thus, modulation of the microbial community structure by GML supplementation was associated with the alleviation of intestinal inflammation in LPS-challenged broilers. In the poultry diet, various feed additives are used to reduce the levels of enteric pathogens by modulating the intestinal microbiome [51], such as xylanase, which enriches gut lactic acid bacteria and reduces the proportion of adverse and pathogenic bacteria [54]. A previous study revealed that GML supplementation attenuated HFD-induced gut microbiota dysbiosis in mice, with increases in Akkermansia, Bifidobacterium, and Lactobacillus abundances [19]. In this study, dietary GML intake increased the abundances of the family Lactobacillaceae and genus Lactobacillus in the cecum of challenged broilers. Lactobacillus can promote gut health as a probiotic bacterium for animals and plays a vital role in maintaining intestinal metabolic capacities and microbial homeostasis [55]. A negative correlation has been widely reported between Lactobacillus and inflammatory cytokines [3]. The increase in the abundance of Lactobacillus due to GML supplementation may explain the depressed intestinal inflammation in LPS-challenged broilers. Administration of GML promoted the colonization of some beneficial bacteria [13], such as the genera Blautia and Coprobacter, in challenged broilers in the present study. Moreover, GML supplementation reversed the reduction in the relative abundances of Anaerostipes, Pseudoflavonifractor, and Gordonibacter induced by LPS challenge. The promoted colonization of probiotics can result in colonization resistance to intestinal pathogens [56], which partly explained the reduced proportions of the family Acidaminococcaceae 
and genera Phascolarctobacterium, Elusimicrobium, and Marvinbryantia in LPS-challenged broilers fed GML-supplied diets. Enhanced adhesion and colonization of probiotics have been shown to result in a defensive resopnse to LPS challenge, which promoted gut health in broiler chicks [3]. In the current study, attenuated inflammation and ameliorated intestinal integrity and antioxidant capacity reduction in LPSchallenged broilers were demonstrated to be positively associated with GML supplementation. Moreover, the augmented immunity, inflammation, intestinal barrier, and antioxidation indices were related to the intestinal microbiota. In mice, dietary GML supplementation has been shown to significantly affect metabolism and immunity through the intestinal microbiota, which considerably impacted host health and physiology [18]. The amelioration of HFD-induced metabolic disorders, obesity, and inflammation by increased GML supplementation may occur through targeting the intestinal microbiota [19]. Mutual regulatory activities exist in a poultry host and its gut microbiota, particularly during the exchange of nutrients and modulation of intestinal morphology, physiology, and immunity [51]. These results indicated that GML supplementation attenuated immunological stress and intestinal mucosal injury mediated by LPS challenge, probably through the alteration of the cecal microbiota.

\section{Conclusions}

Dietary GML supplementation ameliorated LPS-induced immunological stress and intestinal injury in broilers by suppressing inflammation and promoting intestinal barrier function and antioxidant enzyme activity. The alterations in the cecal microbiota modulated by GML supplementation were probably the main reason for the recovery of intestinal health in LPS-challenged broilers. Moreover, the present results confirmed the immunomodulatory, antioxidant, and anti-inflammatory properties of GML in LPS-challenged broilers, which may be associated with the regulation of the AMPK/NF-KB/Nrf2 signaling pathway.

\section{Abbreviations}

GML: glycerol monolaurate; LPS: lipopolysaccharide; NF-kB: nuclear factor kappa-B; AMPK: adenosine monophosphate-activated protein kinase; Nrf2: nuclear factor-erythroid 2-related factor 2; TLR4: toll-like reporter 4; HFD: high-fat diet; AFI: average feed intake; ABWG: average body weight gain; FCR: feed conversion ratio; DAO: diamine oxidase; IL-1 $\beta$ : interleukin 1 beta; TNF-a: tumor necrosis factor-alpha; IFN-Y: interferon-ץ; IgA: immunoglobulin A; VH: villus height; CD: crypt depth; VCR: villus height-to-crypt depth ratio; MDA: malondialdehyde; T-AOC: total antioxidant capacity; CAT: catalase; T-SOD: total superoxide dismutase; OTU: operational taxonomic unit; faith-pd: faith-phylogenetic diversity; PCoA: principal coordinate analysis; LEfSe: Linear discriminant analysis effect size; RDA: redundancy analysis; MIP-3a: macrophage inflammatory protein 3 alpha; ZO-1: zonula occludens; CON: control.

\section{Declarations}

\section{Availability of data and material}

The original contributions presented in the study are publicly available. This data can be found here: http://www.ncbi.nlm.nih.gov/bioproject/774633. 


\section{Ethics approval and consent to participate}

The animal study was reviewed and approved by Ethics Committee of the Shandong Agricultural University.

\section{Authors' contributions}

ZS conceived the idea and provided resources. LK designed the study, performed the experiment, and wrote the manuscript. ZW and ZS provided guidelines. CX and QZ participated in the experiment. All authors contributed to the article and approved the submitted version.

\section{Funding}

This work was supported by the Natural Science Foundation of Shandong Province (ZR2020MC170) and the Shandong Province Agricultural Industry Technology (SDAIT-11-08).

\section{Competing interests}

The authors declare that they have no competing interests.

\section{Consent for publication}

Not applicable.

\section{Author details}

${ }^{1}$ Department of Animal Science and Technology, Shandong Agricultural University, Taian, Shandong 271018, China. ${ }^{2}$ Center for Mitochondria and Healthy Ageing, College of Life Sciences, Yantai University, Yantai, Shandong 264005, China. ${ }^{3}$ Precision Livestock and Nutrition Unit, Gembloux Agro-Bio Tech, University of Liège, Gembloux 5030, Belgium

\section{Acknowledgements}

Not applicable.

\section{References}

1. Surai PF, Fisinin VI. Vitagenes in poultry production: Part 2. Nutritional and internal stresses. World's Poultry Science Journal. 2016;72(4):761-72. https://doi.org/10.1017/S0043933916000726.

2. Connerton PL, Richards PJ, Lafontaine GM, O'Kane PM, Ghaffar N, Cummings NJ et al. The effect of the timing of exposure to Campylobacter jejuni on the gut microbiome and inflammatory responses of broiler chickens. Microbiome. 2018;6(1):88. https://doi.org/10.1186/s40168-018-0477-5.

3. Jiang J, Qi L, Wei Q, Shi F. Maternal stevioside supplementation ameliorates intestinal mucosal damage and modulates gut microbiota in chicken offspring challenged with lipopolysaccharide. Food \& Function. 2021;12(13):6014-28. https://doi.org/10.1039/D0F002871A. 
4. Wang X, Li Y, Shen J, Wang S, Yao J, Yang X. Effect of Astragalus polysaccharide and its sulfated derivative on growth performance and immune condition of lipopolysaccharide-treated broilers. International Journal of Biological Macromolecules. 2015;76:188-94.

https://doi.org/10.1016/j.jibiomac.2015.02.040.

5. Zhang Q, Chen X, Eicher SD, Ajuwon KM, Applegate TJ. Effect of threonine on secretory immune system using a chicken intestinal ex vivo model with lipopolysaccharide challenge. Poultry science. 2017;96(9):3043-51. https://doi.org/10.3382/ps/pex111.

6. Chen Y, Zhang H, Cheng Y, Li Y, Wen C, Zhou Y. Dietary I-threonine supplementation attenuates lipopolysaccharide-induced inflammatory responses and intestinal barrier damage of broiler chickens at an early age. Br J Nutr. 2018;119(11):1254-62. https://doi.org/10.1017/S0007114518000740.

7. Kogut $\mathrm{MH}$. Issues and consequences of using nutrition to modulate the avian immune response. Journal of Applied Poultry Research. 2017;26(4):605-12. https://doi.org/10.3382/japr/pfx028.

8. Amer SA, A-Nasser A, Al-Khalaifah HS, AlSadek DMM, Abdel fattah DM, Roushdy EM et al. Effect of Dietary Medium-Chain a-Monoglycerides on the Growth Performance, Intestinal Histomorphology, Amino Acid Digestibility, and Broiler Chickens' Blood Biochemical Parameters. Animals. 2021;11(1):57. https://doi.org/10.3390/ani11010057.

9. Li Q, Estes JD, Schlievert PM, Duan L, Brosnahan AJ, Southern PJ et al. Glycerol monolaurate prevents mucosal SIV transmission. Nature. 2009;458(7241):1034-8. https://doi.org/10.1038/nature07831.

10. Zhao M, Jiang Z, Cai H, Li Y, Mo Q, Deng L et al. Modulation of the Gut Microbiota during High-Dose Glycerol Monolaurate-Mediated Amelioration of Obesity in Mice Fed a High-Fat Diet. mBio. 2020;11(2):e00190-20. https://doi.org/10.1128/mBio.00190-20.

11. Peterson ML, Schlievert PM. Glycerol Monolaurate Inhibits the Effects of Gram-Positive Select Agents on Eukaryotic Cells. Biochemistry. 2006;45(7):2387-97. https://doi.org/10.1021/bi051992u.

12. Zhang MS, Tran PM, Wolff AJ, Tremblay MM, Fosdick MG, Houtman JCD. Glycerol monolaurate induces filopodia formation by disrupting the association between LAT and SLP-76 microclusters. Science Signaling. 2018;11(528):eaam9095. https://doi.org/10.1126/scisignal.aam9095.

13. Liu T, Tang J, Feng F. Glycerol monolaurate improves performance, intestinal development, and muscle amino acids in yellow-feathered broilers via manipulating gut microbiota. Applied Microbiology and Biotechnology. 2020;104(23):10279-91. https://doi.org/10.1007/s00253-020-10919-y.

14. Diaz Carrasco JM, Casanova NA, Fernández Miyakawa ME. Microbiota, Gut Health and Chicken Productivity: What Is the Connection? Microorganisms. 2019;7(10):374. https://doi.org/10.3390/microorganisms7100374.

15. Kong L, Wang Z, Xiao C, Zhu Q, Song Z. Glycerol Monolaurate Ameliorated Intestinal Barrier and Immunity in Broilers by Regulating Intestinal Inflammation, Antioxidant Balance, and Intestinal Microbiota. Frontiers in Immunology. 2021;12(3913). https://doi.org/10.3389/fimmu.2021.713485.

16. Oakley BB, Lillehoj HS, Kogut MH, Kim WK, Maurer JJ, Pedroso A et al. The chicken gastrointestinal microbiome. FEMS Microbiology Letters. 2014;360(2):100-12. https://doi.org/10.1111/15746968.12608. 
17. Mo Q, Fu A, Deng L, Zhao M, Li Y, Zhang H et al. High-dose Glycerol Monolaurate Up-Regulated Beneficial Indigenous Microbiota without Inducing Metabolic Dysfunction and Systemic Inflammation: New Insights into Its Antimicrobial Potential. Nutrients. 2019;11(9):1981. https://doi.org/10.3390/nu11091981.

18. Jiang Z, Zhao M, Zhang H, Li Y, Liu M, Feng F. Antimicrobial Emulsifier-Glycerol Monolaurate Induces Metabolic Syndrome, Gut Microbiota Dysbiosis, and Systemic Low-Grade Inflammation in Low-Fat Diet Fed Mice. Molecular Nutrition \& Food Research. 2018;62(3):1700547.

https://doi.org/10.1002/mnfr.201700547.

19. Zhao M, Cai H, Jiang Z, Li Y, Zhong H, Zhang H et al. Glycerol-Monolaurate-Mediated Attenuation of Metabolic Syndrome is Associated with the Modulation of Gut Microbiota in High-Fat-Diet-Fed Mice. Molecular Nutrition \& Food Research. 2019;63(18):1801417. https://doi.org/10.1002/mnfr.201801417.

20. Olivier S, Leclerc J, Grenier A, Foretz M, Tamburini J, Viollet B. AMPK Activation Promotes Tight Junction Assembly in Intestinal Epithelial Caco-2 Cells. International Journal of Molecular Sciences. 2019;20(20):5171. https://doi.org/10.3390/ijms20205171.

21. Salminen A, Hyttinen JMT, Kaarniranta K. AMP-activated protein kinase inhibits NF-KB signaling and inflammation: impact on healthspan and lifespan. Journal of Molecular Medicine. 2011;89(7):667-76. https://doi.org/10.1007/s00109-011-0748-0.

22. Yaku K, Matsui-Yuasa I, Konishi Y, Kojima-Yuasa A. AMPK synergizes with the combined treatment of 1'-acetoxychavicol acetate and sodium butyrate to upregulate phase II detoxifying enzyme activities. Molecular Nutrition \& Food Research. 2013;57(7):1198-208. https://doi.org/10.1002/mnfr.201200809.

23. Matzinger M, Fischhuber K, Pölöske D, Mechtler K, Heiss EH. AMPK leads to phosphorylation of the transcription factor Nrf2, tuning transactivation of selected target genes. Redox Biology. 2020;29:101393. https://doi.org/10.1016/j.redox.2019.101393.

24. Fortuoso BF, dos Reis JH, Gebert RR, Barreta M, Griss LG, Casagrande RA et al. Glycerol monolaurate in the diet of broiler chickens replacing conventional antimicrobials: Impact on health, performance and meat quality. Microbial Pathogenesis. 2019;129:161-7. https://doi.org/10.1016/j.micpath.2019.02.005.

25. Kamboh AA, Zhu WY. Individual and combined effects of genistein and hesperidin on immunity and intestinal morphometry in lipopolysacharide-challenged broiler chickens. Poultry science. 2014;93(9):2175-83. https://doi.org/10.3382/ps.2014-03971.

26. Dhabhar FS, Miller AH, McEwen BS, Spencer RL. Effects of stress on immune cell distribution. Dynamics and hormonal mechanisms. The Journal of Immunology. 1995;154(10):5511-27.

27. Pham VH, Kan L, Huang J, Geng Y, Zhen W, Guo Y et al. Dietary encapsulated essential oils and organic acids mixture improves gut health in broiler chickens challenged with necrotic enteritis. J Anim Sci Biotechnol. 2020;11(1):18. https://doi.org/10.1186/s40104-019-0421-y.

28. Lugrin J, Rosenblatt-Velin N, Parapanov R, Liaudet L. The role of oxidative stress during inflammatory processes. Biol Chem. 2014;395(2):203-30. https://doi.org/10.1515/hsz-2013-0241.

29. Coble DJ, Redmond SB, Hale B, Lamont SJ. Distinct lines of chickens express different splenic cytokine profiles in response to Salmonella Enteritidis challenge. Poultry Science. 2011;90(8):1659-63. https://doi.org/10.3382/ps.2010-01279. 
30. Zheng YW, Zhang JY, Zhou HB, Guo YP, Ma QG, Ji C et al. Effects of dietary pyrroloquinoline quinone disodium supplementation on inflammatory responses, oxidative stress, and intestinal morphology in broiler chickens challenged with lipopolysaccharide. Poultry Science. 2020;99(11):5389-98. https://doi.org/10.1016/j.psj.2020.08.007.

31. Jiang J, Qi L, Lv Z, Jin S, Wei X, Shi F. Dietary Stevioside Supplementation Alleviates Lipopolysaccharide-Induced Intestinal Mucosal Damage through Anti-Inflammatory and Antioxidant Effects in Broiler Chickens. Antioxidants. 2019;8(12):575. https://doi.org/10.3390/antiox8120575.

32. Chen Y, Zhang H, Cheng Y, Li Y, Wen C, Zhou Y. Dietary I-threonine supplementation attenuates lipopolysaccharide-induced inflammatory responses and intestinal barrier damage of broiler chickens at an early age. British Journal of Nutrition. 2018;119(11):1254-62. https://doi.org/10.1017/S0007114518000740.

33. Vichaya EG, Hunt SC, Dantzer R. Lipopolysaccharide Reduces Incentive Motivation While Boosting Preference for High Reward in Mice. Neuropsychopharmacology. 2014;39(12):2884-90. https://doi.org/10.1038/npp.2014.141.

34. Groschwitz KR, Hogan SP. Intestinal barrier function: Molecular regulation and disease pathogenesis. Journal of Allergy and Clinical Immunology. 2009;124(1):3-20. https://doi.org/10.1016/j.jaci.2009.05.038.

35. Liu L, Shen J, Zhao C, Wang X, Yao J, Gong Y et al. Dietary Astragalus polysaccharide alleviated immunological stress in broilers exposed to lipopolysaccharide. International Journal of Biological Macromolecules. 2015;72:624-32. https://doi.org/10.1016/j.ijbiomac.2014.08.057.

36. Liu T, Li C, Zhong H, Feng F. Dietary medium-chain a-monoglycerides increase BW, feed intake, and carcass yield in broilers with muscle composition alteration. Poultry Science. 2021;100(1):186-95. https://doi.org/10.1016/j.psj.2020.09.056.

37. Byun E-B, Sung N-Y, Byun E-H, Song D-S, Kim J-K, Park J-H et al. The procyanidin trimer C1 inhibits LPSinduced MAPK and NF-KB signaling through TLR4 in macrophages. International Immunopharmacology. 2013;15(2):450-6. https://doi.org/10.1016/j.intimp.2012.11.021.

38. Sivinski SE, Mamedova LK, Rusk RA, Elrod CC, Swartz TH, McGill JM et al. Development of an macrophage screening system on the immunomodulating effects of feed components. J Anim Sci Biotechnol. 2020;11:89. https://doi.org/10.1186/s40104-020-00497-4.

39. Nishimura Y, Moriyama M, Kawabe K, Satoh H, Takano K, Azuma Y-T et al. Lauric Acid Alleviates Neuroinflammatory Responses by Activated Microglia: Involvement of the GPR40-Dependent Pathway. Neurochem Res. 2018;43(9):1723-35. https://doi.org/10.1007/s11064-018-2587-7.

40. Giri S, Nath N, Smith B, Viollet B, Singh AK, Singh I. 5-Aminoimidazole-4-Carboxamide-1- $\beta$-4Ribofuranoside Inhibits Proinflammatory Response in Glial Cells: A Possible Role of AMP-Activated Protein Kinase. The Journal of Neuroscience. 2004;24(2):479-87. https://doi.org/10.1523/jneurosci.4288-03.2004.

41. Lu J, Wu D-m, Zheng Y-I, Hu B, Zhang Z-f, Shan Q et al. Quercetin activates AMP-activated protein kinase by reducing PP2C expression protecting old mouse brain against high cholesterol-induced neurotoxicity. The Journal of Pathology. 2010;222(2):199-212. https://doi.org/10.1002/path.2754. 
42. Wang W, Li Z, Han Q, Guo Y, Zhang B, D'inca R. Dietary live yeast and mannan-oligosaccharide supplementation attenuate intestinal inflammation and barrier dysfunction induced by Escherichia coli in broilers. British Journal of Nutrition. 2016;116(11):1878-88.

https://doi.org/10.1017/S0007114516004116.

43. Yang L, Liu G, Lian K, Qiao Y, Zhang B, Zhu X et al. Dietary leonurine hydrochloride supplementation attenuates lipopolysaccharide challenge-induced intestinal inflammation and barrier dysfunction by inhibiting the NF-KB/MAPK signaling pathway in broilers. Journal of Animal Science. 2019;97(4):167992. https://doi.org/10.1093/jas/skz078.

44. Zhang B, Guo Y. Supplemental zinc reduced intestinal permeability by enhancing occludin and zonula occludens protein-1 (ZO-1) expression in weaning piglets. Br J Nutr. 2009;102(5):687-93. https://doi.org/10.1017/S0007114509289033.

45. Burlikowska K. DIETARY FRUCTANS AND THEIR POTENTIAL BENEFICIAL INFLUENCE ON HEALTH AND PERFORMANCE PARAMETRS IN BROILER CHICKENS. Journal of Central European Agriculture. 2012;13:272. https://doi.org/10.5513/JCEA01/13.2.1045.

46. Chen L, Wang J, You Q, He S, Meng Q, Gao J et al. Activating AMPK to Restore Tight Junction Assembly in Intestinal Epithelium and to Attenuate Experimental Colitis by Metformin. Frontiers in Pharmacology. 2018;9(761). https://doi.org/10.3389/fphar.2018.00761.

47. Elamin EE, Masclee AA, Dekker J, Pieters H-J, Jonkers DM. Short-Chain Fatty Acids Activate AMPActivated Protein Kinase and Ameliorate Ethanol-Induced Intestinal Barrier Dysfunction in Caco-2 Cell Monolayers. The Journal of Nutrition. 2013;143(12):1872-81. https://doi.org/10.3945/jn.113.179549.

48. Wu QJ, Zhou YM, Wu YN, Zhang LL, Wang T. The effects of natural and modified clinoptilolite on intestinal barrier function and immune response to LPS in broiler chickens. Veterinary Immunology and Immunopathology. 2013;153(1):70-6. https://doi.org/10.1016/j.vetimm.2013.02.006.

49. Zimmermann K, Baldinger J, Mayerhofer B, Atanasov AG, Dirsch VM, Heiss EH. Activated AMPK boosts the Nrf2/HO-1 signaling axis-A role for the unfolded protein response. Free Radical Biology and Medicine. 2015;88:417-26. https://doi.org/10.1016/j.freeradbiomed.2015.03.030.

50. Wang S, Song P, Zou M-H. AMP-activated protein kinase, stress responses and cardiovascular diseases. Clinical Science. 2012;122(12):555-73. https://doi.org/10.1042/cs20110625.

51. Pan D, Yu Z. Intestinal microbiome of poultry and its interaction with host and diet. Gut Microbes. 2014;5(1):108-19. https://doi.org/10.4161/gmic.26945.

52. Lucke A, Böhm J, Zebeli Q, Metzler-Zebeli BU. Dietary Deoxynivalenol Contamination and Oral Lipopolysaccharide Challenge Alters the Cecal Microbiota of Broiler Chickens. Frontiers in Microbiology. 2018;9(804). https://doi.org/10.3389/fmicb.2018.00804.

53. Ott SJ, Musfeldt M, Wenderoth DF, Hampe J, Brant O, Fölsch UR et al. Reduction in diversity of the colonic mucosa associated bacterial microflora in patients with active inflammatory bowel disease. Gut. 2004;53(5):685-93. https://doi.org/10.1136/gut.2003.025403.

54. Rodríguez ML, Rebolé A, Velasco S, Ortiz LT, Treviño J, Alzueta C. Wheat- and barley-based diets with or without additives influence broiler chicken performance, nutrient digestibility and intestinal microflora. J Sci Food Agric. 2012;92(1):184-90. https://doi.org/10.1002/jsfa.4561. 
55. Angelakis E. Weight gain by gut microbiota manipulation in productive animals. Microbial Pathogenesis. 2017;106:162-70. https://doi.org/10.1016/j.micpath.2016.11.002.

56. Imhann F, Bonder MJ, Vich Vila A, Fu J, Mujagic Z, Vork L et al. Proton pump inhibitors affect the gut microbiome. Gut. 2016;65(5):740-8. http://doi.org/10.1136/gutjnl-2015-310376.

\section{Tables}

Table 1 Effects of glycerol monolaurate supplementation on the growth performance of lipopolysaccharidechallenged broilers

\begin{tabular}{llllllllll} 
Item & \multicolumn{2}{l}{ Treatment } & \multicolumn{5}{c}{ SEM } & \multicolumn{2}{l}{$P$-value } \\
\cline { 2 - 3 } & CON & GML & LPS & LPS + GML & & GML & LPS & Interaction \\
\hline AFI (g/bird) & 477.06 & 469.14 & 448.68 & 450.29 & 3.76 & 0.684 & 0.016 & 0.541 \\
\hline ABWG (g/bird) & 345.91 & 340.30 & 313.60 & 312.81 & 4.32 & 0.805 & 0.002 & 0.819 \\
\hline FCR & 1.37 & 1.36 & 1.43 & 1.44 & 0.01 & 0.827 & 0.021 & 0.695
\end{tabular}

$>$

$A F I$, average feed intake; $A B W G$, average body weight gain; FCR, feed conversion ratio, defined as AFI/ABWG.

Values are expressed as least squares means $\pm \operatorname{SEMs}(n=6)$.

Table 2 Effects of glycerol monolaurate supplementation on the blood cell counts of lipopolysaccharidechallenged broilers

\begin{tabular}{lllllllll} 
Item & \multicolumn{2}{l}{ Treatment } & \multicolumn{7}{c}{ SEM } & $P$-value \\
\hline$\left(10^{9}\right.$ cells $\left./ \mathrm{l}\right)$ & CON & GML & LPS & LPS + GML & & GML & LPS & Interaction \\
\hline Leukocytes & 85.81 & 72.28 & 87.43 & 81.03 & 1.11 & $<0.001$ & 0.025 & 0.118 \\
\hline Lymphocytes & 65.46 & 61.83 & 65.02 & 60.46 & 1.65 & 0.223 & 0.787 & 0.889 \\
\hline Intermediate cells & 6.84 & 6.30 & 7.47 & 7.74 & 0.14 & 0.649 & 0.001 & 0.162 \\
\hline Granulocytes & 9.77 & 7.22 & 11.51 & 11.17 & 0.38 & 0.070 & 0.001 & 0.115
\end{tabular}

Values are expressed as least squares means \pm SEMs $(n=12)$.

Table 3 Effects of glycerol monolaurate supplementation on immune indicators in the sera of lipopolysaccharide-challenged broilers 


\begin{tabular}{|c|c|c|c|c|c|c|c|c|}
\hline \multirow[t]{2}{*}{ Item } & \multicolumn{4}{|l|}{ Treatment } & \multirow[t]{2}{*}{ SEM } & \multicolumn{3}{|l|}{$P$-value } \\
\hline & CON & GML & LPS & LPS+GML & & GML & LPS & Interaction \\
\hline $\begin{array}{l}\mathrm{IL}-1 \beta \\
(\mathrm{pg} / \mathrm{ml})\end{array}$ & 637.90 & 599.65 & 660.78 & 645.94 & 6.05 & 0.040 & 0.010 & 0.345 \\
\hline $\begin{array}{l}\mathrm{IL}-6 \\
(\mathrm{pg} / \mathrm{ml})\end{array}$ & $25.71^{b}$ & $29.18^{b}$ & $40.78^{a}$ & $30.76^{b}$ & 1.62 & 0.323 & 0.016 & 0.047 \\
\hline $\begin{array}{l}\text { TNF-a } \\
(\mathrm{pg} / \mathrm{ml})\end{array}$ & 62.86 & 58.26 & 63.42 & 66.00 & 0.55 & 0.371 & 0.002 & 0.419 \\
\hline $\begin{array}{l}\text { IFN- } \\
\mathrm{Y}(\mathrm{pg} / \mathrm{ml})\end{array}$ & $60.93^{b}$ & $60.73^{b}$ & $70.77^{a}$ & $64.32^{\mathrm{b}}$ & 1.09 & 0.004 & $<0.001$ & 0.007 \\
\hline $\begin{array}{l}\operatorname{lgA} \\
(\mathrm{pg} / \mathrm{ml})\end{array}$ & $223.83^{b}$ & $231.83^{b}$ & $229.33^{b}$ & $255.75^{a}$ & 1.68 & $<0.001$ & $<0.001$ & 0.009 \\
\hline $\begin{array}{l}\lg G \\
(\mathrm{pg} / \mathrm{ml})\end{array}$ & $1846.11^{b}$ & $1740.56^{b}$ & $1777.22^{b}$ & $2066.67^{a}$ & 25.18 & 0.076 & 0.015 & $<0.001$ \\
\hline
\end{tabular}

IL-1 $\beta$, interleukin 1 $\beta$; IL-6, interleukin 6; TNF-a, tumor necrosis factor $\alpha$; IFN- $\gamma$, interferon $\gamma$; IgA, immunoglobulin $A ;$ IgG, immunoglobulin $G$.

Values are expressed as least squares means \pm SEMs $(n=12)$.

$a, b$ Different superscripts in the same line indicate significant differences according to Tukey's multiple comparisons $(P<0.05)$.

Table 4 Effects of glycerol monolaurate supplementation on inflammatory gene expression in the jejunum of lipopolysaccharide-challenged broilers 


\begin{tabular}{|c|c|c|c|c|c|c|c|c|}
\hline \multirow[t]{2}{*}{ Gene } & \multicolumn{4}{|c|}{ Treatment } & \multirow[t]{2}{*}{ SEM } & \multicolumn{3}{|c|}{$P$-value } \\
\hline & CON & GML & LPS & LPS+GML & & GML & LPS & Interaction \\
\hline$I L-1 \beta$ & 1.00 & 1.15 & 1.20 & 1.01 & 0.05 & 0.861 & 0.304 & 0.585 \\
\hline IL-6 & $1.00^{\mathrm{b}}$ & $1.34^{\mathrm{ab}}$ & $1.69^{a}$ & $0.98^{b}$ & 0.08 & 0.289 & 0.346 & 0.010 \\
\hline$T N F-a$ & 1.00 & 1.13 & 1.12 & 1.07 & 0.06 & 0.873 & 0.716 & 0.683 \\
\hline$I F N-y$ & 1.00 & 0.64 & 1.07 & 0.91 & 0.09 & 0.158 & 0.342 & 0.603 \\
\hline MIP-3a & $1.00^{\mathrm{b}}$ & $0.94^{b}$ & $1.67^{a}$ & $0.96^{\mathrm{b}}$ & 0.06 & 0.016 & 0.018 & 0.024 \\
\hline TLR4 & $1.00^{\mathrm{b}}$ & $1.26^{\mathrm{ab}}$ & $1.95^{\mathrm{a}}$ & $1.06^{b}$ & 0.10 & 0.144 & 0.083 & 0.012 \\
\hline$I K B a$ & 1.00 & 1.09 & 0.76 & 0.90 & 0.05 & 0.266 & 0.044 & 0.795 \\
\hline$N F-K B$ & $1.00^{\mathrm{b}}$ & $1.20^{\mathrm{b}}$ & $1.61^{\mathrm{a}}$ & $0.98^{\mathrm{b}}$ & 0.04 & 0.020 & 0.033 & 0.001 \\
\hline Caspase-1 & $1.00^{\mathrm{b}}$ & $0.97^{b}$ & $1.51^{\mathrm{a}}$ & $1.09^{b}$ & 0.05 & 0.052 & 0.001 & 0.023 \\
\hline
\end{tabular}

IL-1 $\beta$, interleukin 1 $\beta$, IL-6, interleukin 6; TNF-a, tumor necrosis factor $a$, IFN- $\gamma$, interferon $\gamma$; MIP-3a, macrophage inflammatory protein-3a; TLR4, toll-like receptor; ІкBa, inhibitor kappa-B $\alpha$; NF-kB, nuclear factor kappa-B.

Values are expressed as least squares means \pm SEMs $(n=6)$.

a,b Different superscripts in the same line indicate significant differences according to Tukey's multiple comparisons $(P<0.05)$.

Table 5 Effects of glycerol monolaurate supplementation on jejunal barrier function and AMPK levels in lipopolysaccharide-challenged broilers 


\begin{tabular}{|c|c|c|c|c|c|c|c|c|}
\hline \multirow[t]{2}{*}{ Item } & \multicolumn{4}{|c|}{ Treatment } & \multirow[t]{2}{*}{ SEM } & \multicolumn{3}{|c|}{$P$-value } \\
\hline & CON & GML & LPS & LPS+GML & & GML & LPS & Interaction \\
\hline $\mathrm{DAO}(\mathrm{ng} / \mathrm{ml})$ & 26.87 & 29.26 & 36.31 & 32.57 & 1.41 & 0.813 & 0.029 & 0.283 \\
\hline LPS (EU/ml) & $88.64^{b}$ & $92.41^{\mathrm{ab}}$ & $95.83^{a}$ & $91.77^{b}$ & 0.65 & 0.607 & 0.017 & 0.031 \\
\hline $\mathrm{VH}(\mu \mathrm{m})$ & $799.00^{a}$ & $805.50^{a}$ & $669.80^{b}$ & $790.38^{a}$ & 12.59 & 0.017 & 0.007 & 0.030 \\
\hline $\mathrm{CD}(\mu \mathrm{m})$ & 71.20 & 57.55 & 70.43 & 62.25 & 1.59 & 0.001 & 0.539 & 0.393 \\
\hline $\operatorname{VCR}(\mu \mathrm{m})$ & 11.53 & 14.39 & 9.63 & 12.35 & 0.40 & 0.004 & 0.029 & 0.934 \\
\hline $\begin{array}{l}\text { p-AMPK } \\
\text { (ng/mgprot) }\end{array}$ & $14.20^{\mathrm{ab}}$ & $14.59^{\mathrm{ab}}$ & $12.72^{\mathrm{b}}$ & $15.64^{a}$ & 0.26 & 0.003 & 0.682 & 0.021 \\
\hline $\begin{array}{l}\text { AMPK } \\
\text { (ng/mgprot) }\end{array}$ & $16.39^{\mathrm{ab}}$ & $15.47^{b}$ & $14.22^{b}$ & $18.14^{\mathrm{a}}$ & 0.32 & 0.024 & 0.691 & 0.001 \\
\hline
\end{tabular}

DAO, diamine oxidase; LPS, lipopolysaccharide; VH, villus height; $C D$, crypt depth; $V C R$, villus height-to-crypt depth ratio; p-AMPK, phospho-adenosine monophosphate-activated protein kinase; AMPK, adenosine monophosphate-activated protein kinase; mgprot, milligrams of protein.

Values are expressed as least squares means \pm SEMs $(n=12)$.

a,b Different superscripts in the same line indicate significant differences according to Tukey's multiple comparisons $(P<0.05)$.

Table 6 Effects of glycerol monolaurate supplementation on gene expression of tight junction protein and AMPK

\begin{tabular}{|c|c|c|c|c|c|c|c|c|}
\hline \multirow[t]{2}{*}{ Gene } & \multicolumn{4}{|c|}{ Treatment } & \multirow[t]{2}{*}{ SEM } & \multicolumn{3}{|c|}{$P$-value } \\
\hline & CON & GML & LPS & LPS+GML & & GML & LPS & Interaction \\
\hline$Z O-1$ & $1.00^{\mathrm{a}}$ & $0.93^{a}$ & $0.60^{b}$ & $0.99^{a}$ & 0.07 & 0.555 & 0.087 & 0.039 \\
\hline occludin & $1.00^{\mathrm{a}}$ & $0.90^{\mathrm{ab}}$ & $0.67^{b}$ & $0.86^{a b}$ & 0.03 & 0.467 & 0.01 & 0.027 \\
\hline claudin-1 & 1.00 & 0.82 & 0.56 & 0.92 & 0.06 & 0.507 & 0.205 & 0.057 \\
\hline claudin-2 & $1.00^{\mathrm{a}}$ & $0.81^{a b}$ & $0.31^{\mathrm{b}}$ & $0.75^{\mathrm{ab}}$ & 0.07 & 0.376 & 0.017 & 0.039 \\
\hline claudin-3 & 1.00 & 1.09 & 1.04 & 1.37 & 0.03 & 0.134 & 0.243 & 0.358 \\
\hline AMPKa1 & $1.00^{\mathrm{a}}$ & $0.67^{a b}$ & $0.48^{\mathrm{b}}$ & $0.66^{a b}$ & 0.06 & 0.560 & 0.038 & 0.046 \\
\hline AMPКа2 & 1.00 & 1.20 & 0.63 & 0.98 & 0.07 & 0.053 & 0.039 & 0.561 \\
\hline
\end{tabular}

ZO-1, zonula occludens 1; AMPKa, adenosine monophosphate-activated protein kinase $a$. 
Values are expressed as least squares means $\pm \operatorname{SEMs}(n=6)$.

a,b Different superscripts in the same line indicate significant differences according to Tukey's multiple comparisons $(P<0.05)$.

Table 7 Effects of glycerol monolaurate supplementation on the oxidative status in lipopolysaccharidechallenged broilers

Item

Treatment

SEM $P$-value

CON GML LPS LPS+GML GML LPS Interaction

Serum

\begin{tabular}{|c|c|c|c|c|c|c|c|c|}
\hline MDA (nmol/ml) & $4.86^{b}$ & $5.03^{b}$ & $7.76^{\mathrm{a}}$ & $5.19^{b}$ & 0.33 & 0.077 & 0.027 & 0.046 \\
\hline $\mathrm{SOD}(\mathrm{ng} / \mathrm{ml})$ & 7.80 & 7.66 & 7.57 & 7.91 & 0.18 & 0.953 & 0.587 & 0.173 \\
\hline CAT (pg/ml) & $2.64^{a}$ & $2.41^{\mathrm{ab}}$ & $1.77^{b}$ & $2.57^{a}$ & 0.11 & 0.232 & 0.138 & 0.035 \\
\hline $\begin{array}{l}\text { T-AOC } \\
(\mathrm{mmol} / \mathrm{ml})\end{array}$ & $0.52^{\mathrm{a}}$ & $0.45^{\mathrm{ab}}$ & $0.37^{b}$ & $0.49^{a}$ & 0.02 & 0.523 & 0.14 & 0.026 \\
\hline GSH-px (ng/ml) & $147.47^{b}$ & $143.63^{b}$ & $143.42^{b}$ & $156.3^{\mathrm{a}}$ & 8.03 & 0.029 & 0.037 & $<0.001$ \\
\hline \multicolumn{9}{|l|}{ Jejunum } \\
\hline $\begin{array}{l}\text { MDA } \\
\text { (nmol/mgprot) }\end{array}$ & 28.37 & 29.12 & 64.03 & 41.63 & 3.98 & 0.186 & 0.006 & 0.158 \\
\hline $\begin{array}{l}\text { SOD } \\
\text { (ng/mgprot) }\end{array}$ & $1.75^{b}$ & $1.78^{\mathrm{ab}}$ & $1.61^{b}$ & $2.01^{a}$ & 0.03 & 0.002 & 0.478 & 0.006 \\
\hline $\begin{array}{l}\text { CAT } \\
\text { (pg/mgprot) }\end{array}$ & $12.52^{b}$ & $12.01^{\mathrm{ab}}$ & $10.93^{b}$ & $14.33^{\mathrm{a}}$ & 0.26 & 0.012 & 0.492 & 0.001 \\
\hline $\begin{array}{l}\text { T-AOC } \\
\text { (nmol/mgprot) }\end{array}$ & $9.29^{a}$ & $9.87^{\mathrm{a}}$ & $6.79^{b}$ & $9.54^{a}$ & 0.25 & 0.002 & 0.008 & 0.038 \\
\hline $\begin{array}{l}\text { GSH-px } \\
\text { (ng/mgprot) }\end{array}$ & $38.49^{a b}$ & $35.51^{\mathrm{b}}$ & $34.49^{b}$ & $43.28^{a}$ & 0.66 & 0.035 & 0.164 & $<0.001$ \\
\hline
\end{tabular}

MDA, malondialdehyde; SOD, superoxide dismutase; CAT, catalase; T-AOC, total antioxidant capacity; GSH$\mathrm{px}$, glutathione peroxidase; mgprot, milligrams of protein.

Values are expressed as least squares means $\pm \operatorname{SEMs~}(n=12)$.

a,b Different superscripts in the same line indicate significant differences according to Tukey's multiple comparisons $(P<0.05)$. 
Table 8 Effects of glycerol monolaurate supplementation on antioxidant gene expression in the jejunum of lipopolysaccharide-challenged broilers

\begin{tabular}{|c|c|c|c|c|c|c|c|c|}
\hline \multirow[t]{2}{*}{ Gene } & \multicolumn{4}{|c|}{ Treatment } & \multirow[t]{2}{*}{ SEM } & \multicolumn{3}{|c|}{$P$-value } \\
\hline & CON & GML & LPS & LPS+GML & & GML & LPS & Interaction \\
\hline Nrf2 & $1.00^{\mathrm{a}}$ & $0.83^{a}$ & $0.40^{\mathrm{b}}$ & $0.65^{\mathrm{ab}}$ & 0.05 & 0.684 & 0.002 & 0.046 \\
\hline $\mathrm{HO}-1$ & 1.00 & 1.37 & 0.99 & 1.28 & 0.06 & 0.023 & 0.710 & 0.722 \\
\hline SOD1 & $1.00^{\mathrm{a}}$ & $0.91^{\mathrm{a}}$ & $0.53^{b}$ & $0.95^{a}$ & 0.05 & 0.163 & 0.075 & 0.041 \\
\hline SOD2 & 1.00 & 0.89 & 0.66 & 0.78 & 0.05 & 0.992 & 0.020 & 0.194 \\
\hline CAT & 1.00 & 1.26 & 0.58 & 0.77 & 0.08 & 0.159 & 0.011 & 0.849 \\
\hline GPX1 & 1.00 & 1.06 & 1.04 & 1.07 & 0.07 & 0.937 & 0.650 & 0.632 \\
\hline
\end{tabular}

Nrf2, nuclear factor-erythroid 2-related factor 2; HO-1, heme oxygenase-1; SOD1, superoxide dismutase-1; SOD2, superoxide dismutase-2; CAT, catalase; GPX1, glutathione peroxidase 1.

Values are expressed as least squares means \pm SEMs $(n=12)$.

a,b Different superscripts in the same line indicate significant differences according to Tukey's multiple comparisons $(P<0.05)$.

\section{Figures}



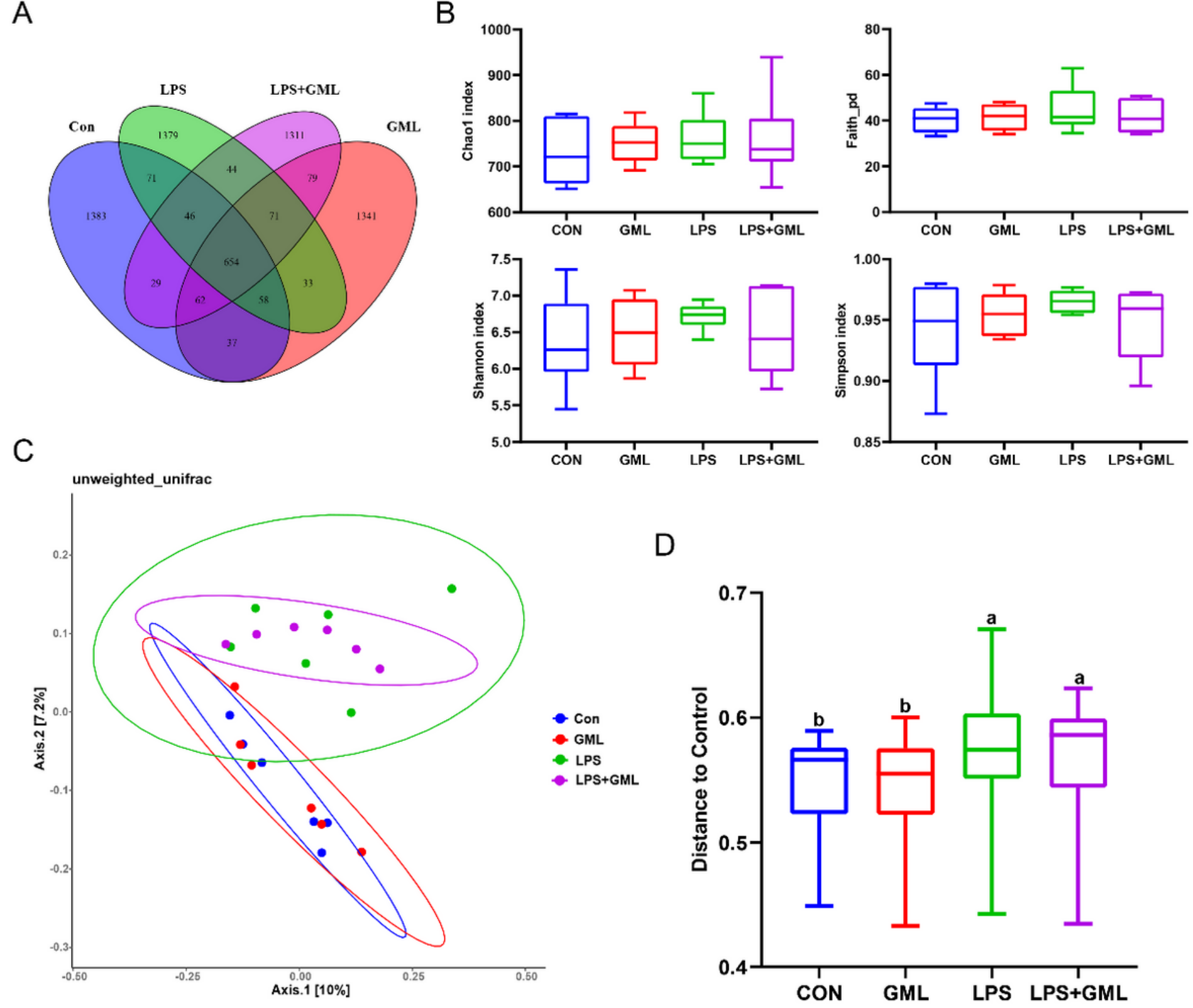

\section{Figure 1}

GML supplementation altered the composition and community diversity of the cecal microbiota in LPSchallenged broilers. (A) Venn diagram for the treatments at the OTU level. (B) a diversity based on the Chao1, Faith_pd, Shannon, and Simpson indices. (C) PCoA based on unweighted UniFrac distance. (D) ANOSIM based on unweighted UniFrac distance; different superscripts indicate significant differences $(\mathrm{P}<$ 0.05). CON, basal diet and injection with saline; GML, basal diet supplemented with $1200 \mathrm{mg} / \mathrm{kg}$ GML and injection with saline; LPS, basal diet and injection with $1 \mathrm{mg} / \mathrm{kg}$ body weight E. coli LPS; LPS + GML, 1200 $\mathrm{mg} / \mathrm{kg}$ GML-supplemented diet and injection with $1 \mathrm{mg} / \mathrm{kg}$ body weight E. coli LPS. 
A

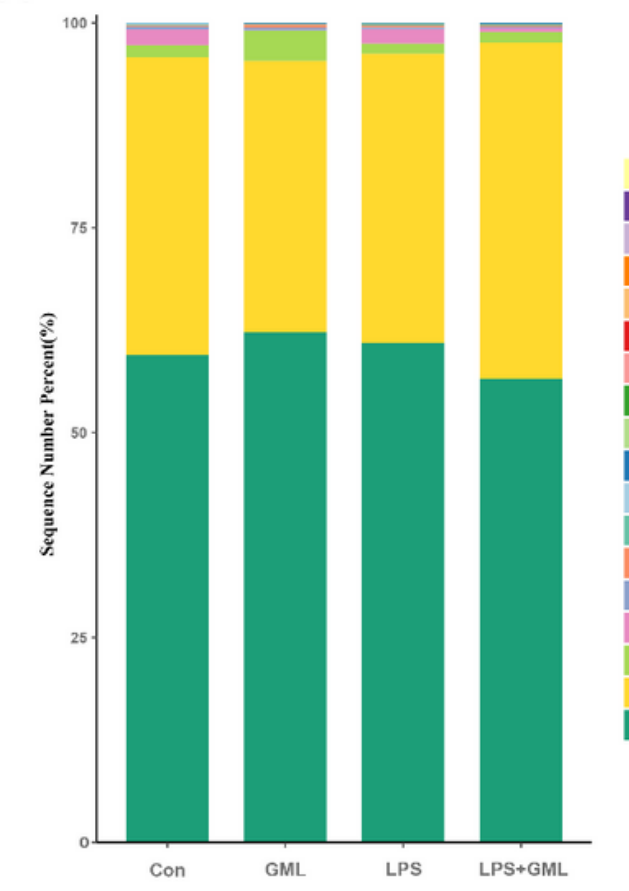

C

Elusimicrobia phylum

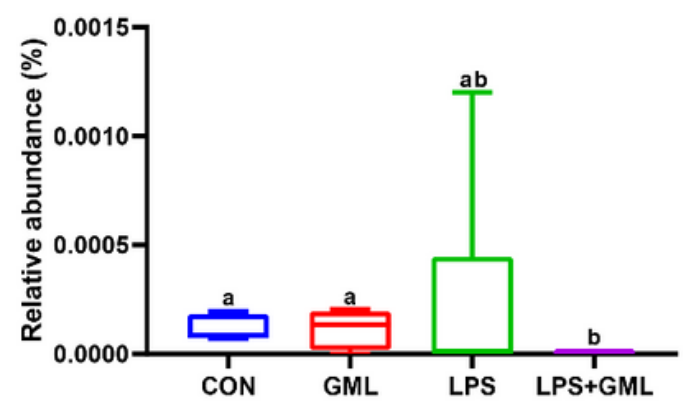

Lactobacillaceae family

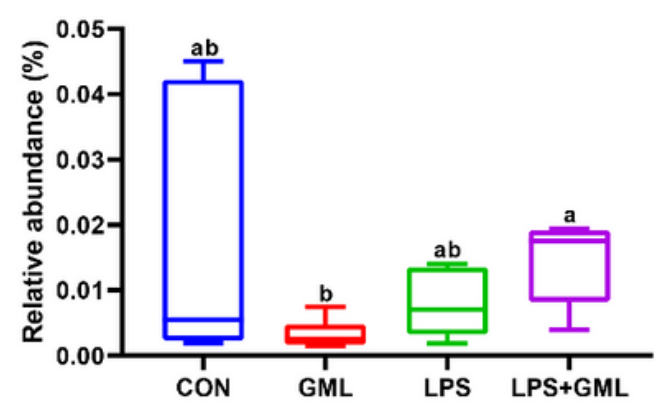

B

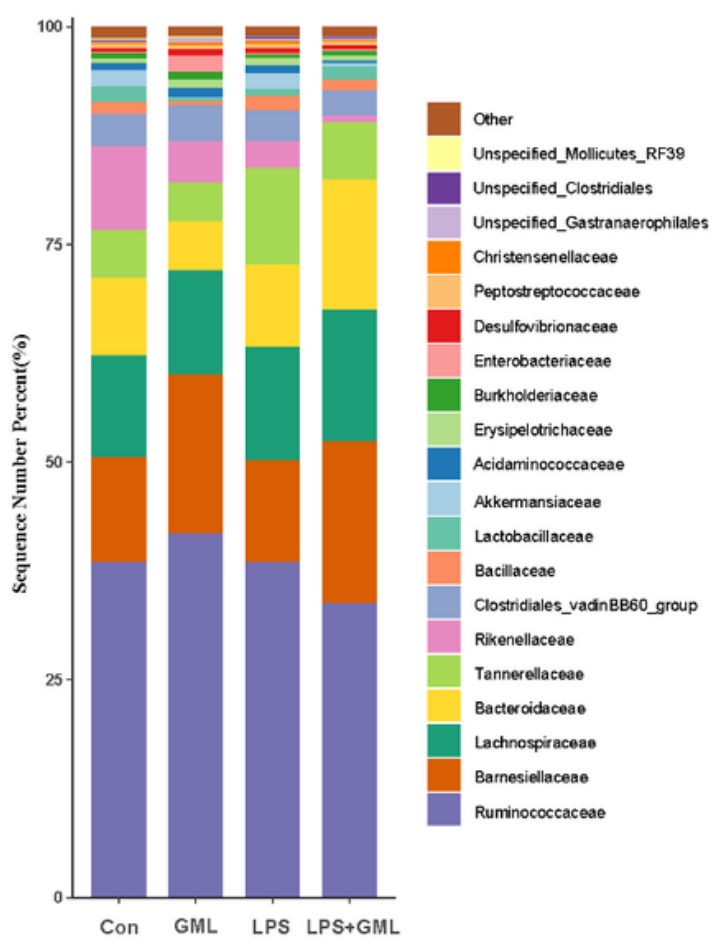

Elusimicrobiaceae family

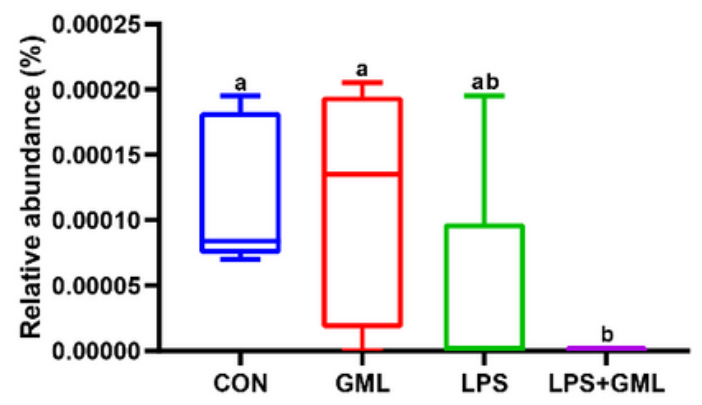

Acidaminococcaceae family

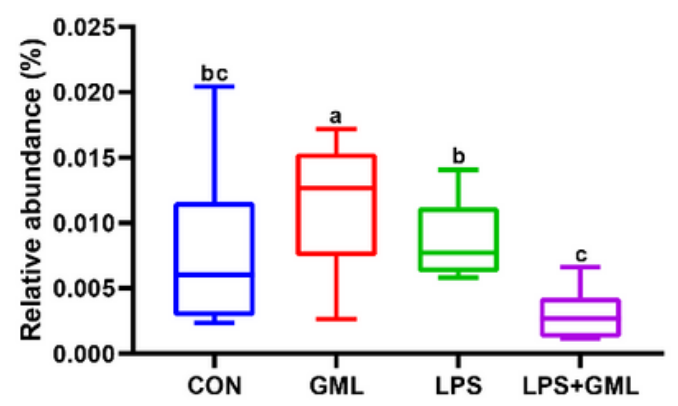

Figure 2

Alteration of the cecal microbiota at the phylum and family levels. (A) The top 20 phyla based on relative abundance in each group. (B) The top 20 families based on relative abundance in each group. (C) Relative abundances of the Elusimicrobia phylum and Elusimicrobiaceae, Lactobacillaceae, and Acidaminococcaceae families. Different superscripts indicate significant differences according to the Kruskal-Wallis test $(P<0.05)$. CON, basal diet and injection with saline; $G M L$, basal diet supplemented with 

LPS; LPS + GML, 1200 mg/kg GML-supplemented diet and injection with 1 mg/kg body weight E. coli LPS.

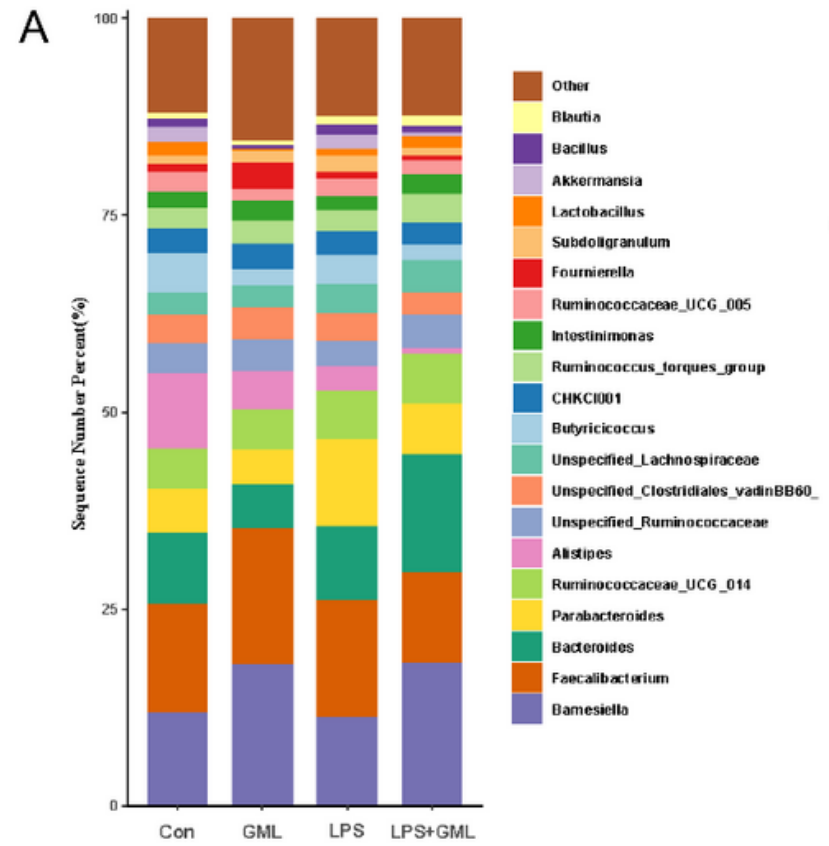

C
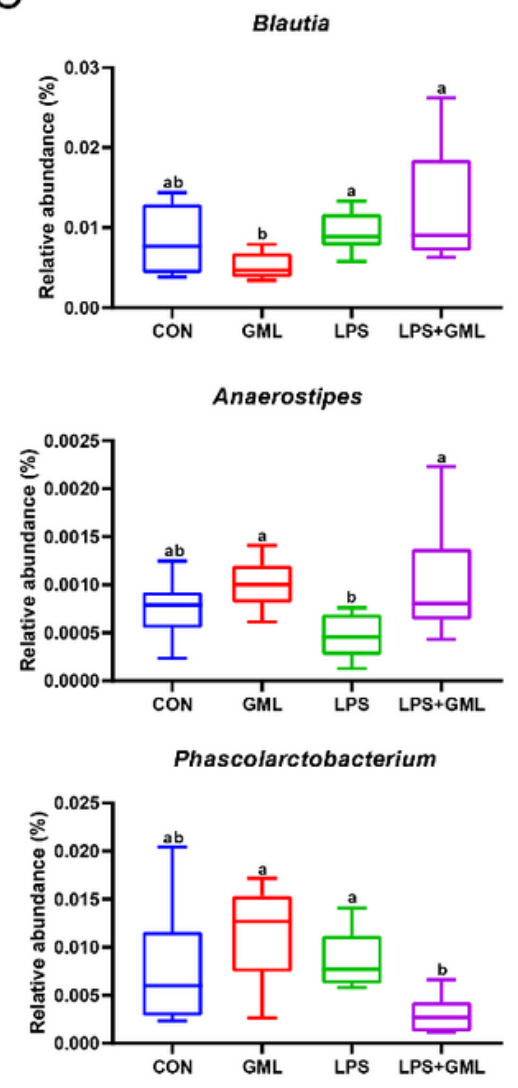

B

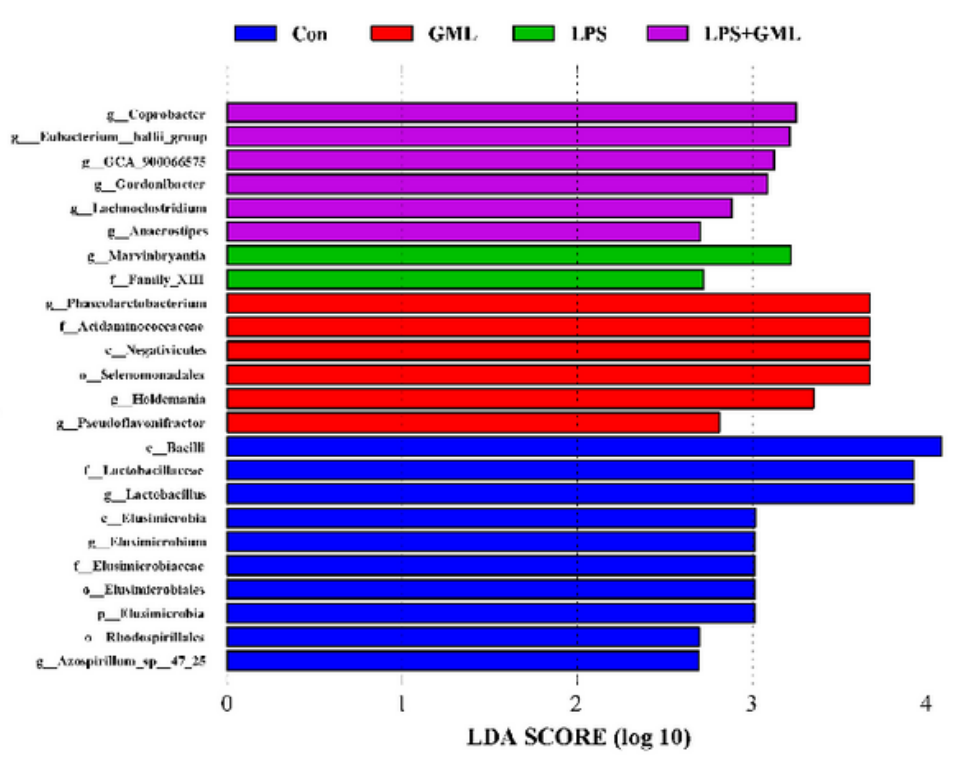

Lactobacillus

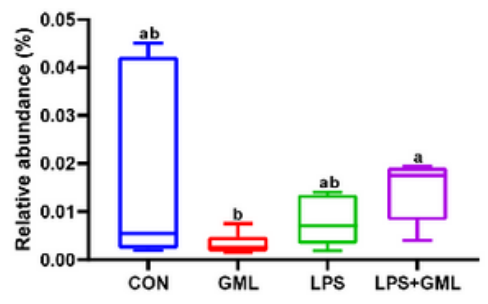

Pseudoflavonifractor
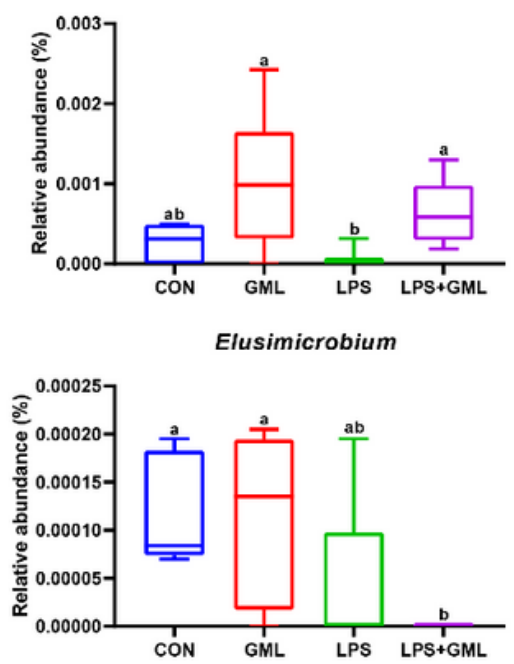

Coprobacter

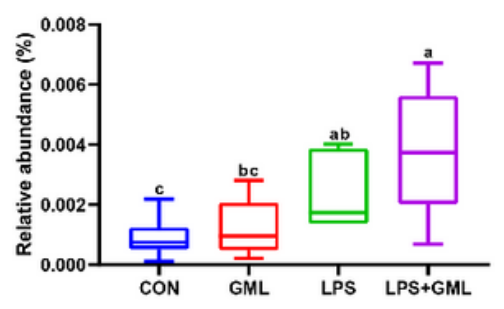

Gordonibacter
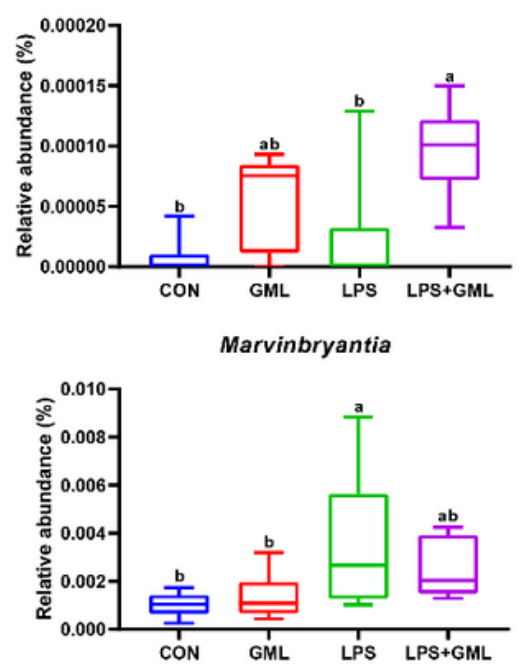

\section{Figure 3}

Alteration of the cecal microbiota at the genus level. (A) Relative abundances of the top 20 bacterial genera present in each group. (B) LEfSe of the cecal microbiota (LDA score greater than 2). (C) The relative abundances differ significantly among groups at the genus level. Different superscripts indicate significant differences according to the Kruskal-Wallis test $(P<0.05)$. CON, basal diet and injection with saline; GML, 
basal diet supplemented with $1200 \mathrm{mg} / \mathrm{kg}$ GML and injection with saline; LPS, basal diet and injection with $1 \mathrm{mg} / \mathrm{kg}$ body weight E. coli LPS; LPS + GML, $1200 \mathrm{mg} / \mathrm{kg}$ GML-supplemented diet and injection with 1 $\mathrm{mg} / \mathrm{kg}$ body weight E. coli LPS.

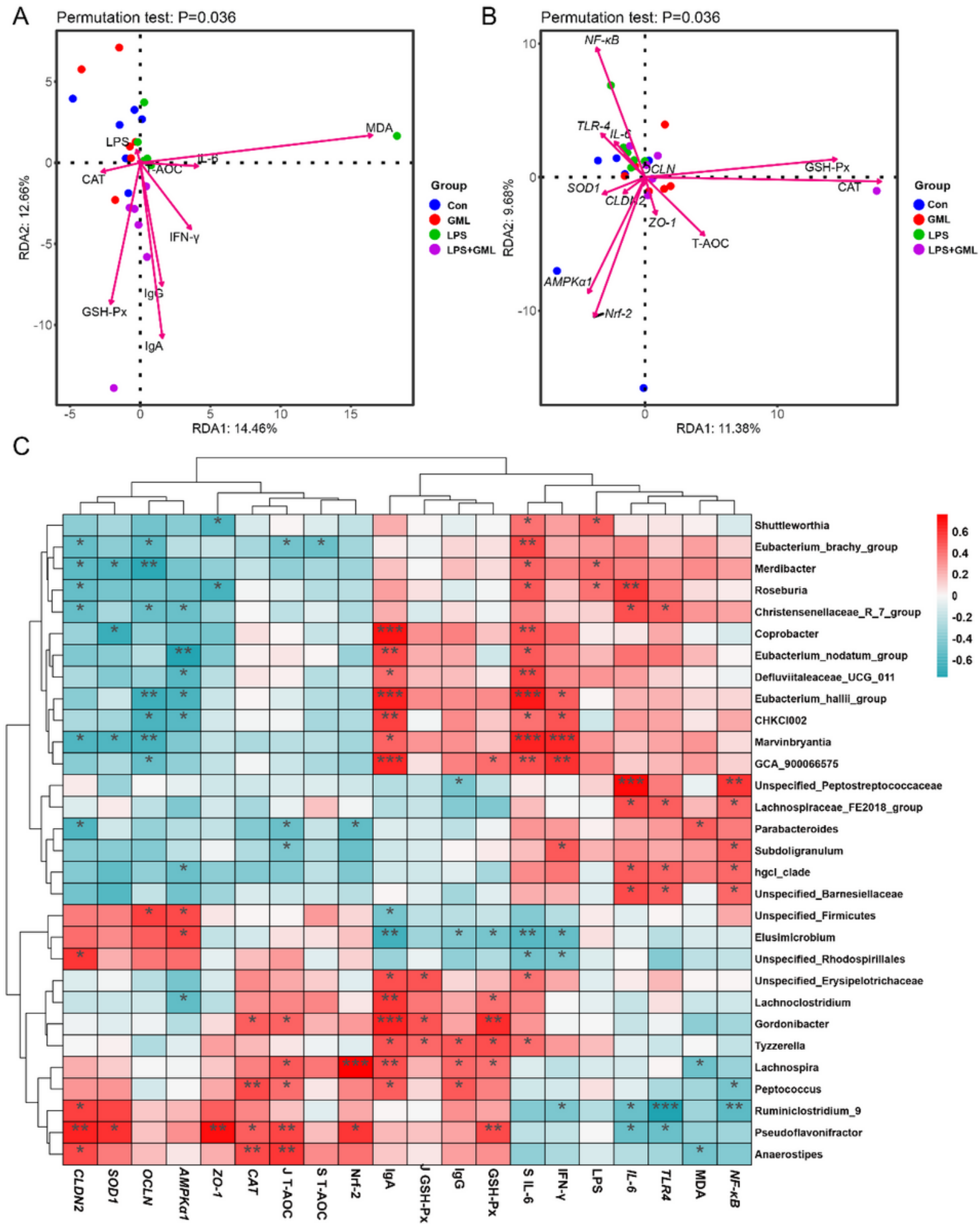

Figure 4

The correlation between serum (A) and jejunal (B) factors and sample distribution by redundancy analysis. (C) Heatmap of correlations determined by Spearman analysis among the cecal microbiota, inflammatory factors, tight junctions, antioxidant enzymes, and intestinal barrier parameters. ${ }^{*} \mathrm{P}<0.05$, ${ }^{\star *} \mathrm{P}<0.01$, ${ }^{\star \star \star} \mathrm{P}<$ 
0.001. CON, basal diet and injection with saline; GML, basal diet supplemented with $1200 \mathrm{mg} / \mathrm{kg} \mathrm{GML}$ and injection with saline; LPS, basal diet and injection with $1 \mathrm{mg} / \mathrm{kg}$ body weight E. coli LPS; LPS + GML, 1200 $\mathrm{mg} / \mathrm{kg}$ GML-supplemented diet and injection with $1 \mathrm{mg} / \mathrm{kg}$ body weight E. coli LPS.

\section{Supplementary Files}

This is a list of supplementary files associated with this preprint. Click to download.

- SupplementaryTableS1.docx

- SupplementaryTableS2.docx 